\title{
An Assessment of Computational Methods for Calculating Accurate Non- Covalent Interaction Energies in 1,2,3,5-Dithiadiazolyl Radicals
}

\author{
Sahar Nikoo and Jeremy M. Rawson* \\ Department of Chemistry and Biochemistry, University of Windsor, Windsor, Ontario, N9B 3P4, \\ Canada
}

\section{Supporting Information}

SUP-1 Total Interaction Energy Computations.

SUP-2 QTAIM Parameters at BCPs and RCPs. 


\section{SUP-1 Total interaction energies of Int.1-X (X = F, Cl, Br) - Int.6-CN in Figure 3 (Table S1-S15).}

Table S1. CP-corrected and uncorrected interaction energy for Int.1-Cl $\left(\mathrm{kJ} \mathrm{mol}^{-1}\right)$ as a function of method and basis set. The benchmark $\operatorname{CCSD}(\mathrm{T}) / \mathrm{CBS}$ value for this interaction is $-20.5 \mathrm{~kJ} \mathrm{~mol}^{-1}$.

\begin{tabular}{|c|c|c|c|c|c|c|c|c|c|c|c|c|c|c|c|c|}
\hline \multirow{2}{*}{$\begin{array}{l}\text { Method } \\
\text { Basis Set }\end{array}$} & \multicolumn{2}{|c|}{ B3LYP-D3 } & \multicolumn{2}{|c|}{ CAM-B3LYP-D3 } & \multicolumn{2}{|c|}{ LC- $\omega$ PBE-D3 } & \multicolumn{2}{|c|}{$\omega B 97 X D$} & \multicolumn{2}{|c|}{ M06-2X } & \multicolumn{2}{|c|}{ M11 } & \multicolumn{2}{|c|}{ BLYP-D3 } & \multicolumn{2}{|c|}{ PBEPBE-D3 } \\
\hline & Uncorr. & Corr. & Uncorr. & Corr. & Uncorr. & Corr. & Uncorr. & Corr. & Uncorr. & Corr. & Uncorr. & Corr. & Uncorr & Corr. & Uncorr & Corr. \\
\hline $6-311 G(d)$ & -22.8 & -14.6 & -25.1 & -16.8 & -24.6 & -16.6 & -18.6 & -11.0 & -26.8 & -19.0 & -23.9 & -16.0 & -20.8 & -12.2 & -23.1 & -14.8 \\
\hline $6-311+G(d)$ & -19.6 & -14.2 & -22.1 & -16.6 & -22.6 & -16.5 & -16.7 & -11.0 & -25.3 & -19.5 & -23.1 & -16.5 & -17.1 & -11.8 & -20.2 & -14.5 \\
\hline $6-311 G(2 d)$ & -18.7 & -12.3 & -20.5 & -13.9 & -19.5 & -13.4 & -14.7 & -8.9 & -21.6 & -15.5 & -18.0 & -12.0 & -17.3 & -10.6 & -19.4 & -13.1 \\
\hline $6-311+G(2 d)$ & -15.0 & -12.4 & -16.8 & -14.1 & -16.7 & -13.8 & -12.1 & -9.3 & -19.2 & -16.5 & -16.7 & -12.9 & -13.1 & -10.5 & -15.8 & -13.1 \\
\hline $6-311 G(d f)$ & -22.8 & -14.3 & -25.2 & -16.5 & -24.5 & -16.2 & -18.7 & -10.7 & -26.7 & -18.6 & -23.7 & -15.4 & -20.8 & -12.0 & -23.1 & -14.5 \\
\hline $6-311+G(d f)$ & -19.7 & -14.0 & -22.2 & -16.4 & -22.4 & -16.2 & -16.7 & -10.8 & -25.2 & -19.2 & -23.0 & -16.1 & -17.1 & -11.6 & -20.2 & -14.2 \\
\hline $6-311 G(2 d f)$ & -18.9 & -12.4 & -20.7 & -14.1 & -19.6 & -13.4 & -14.8 & -8.9 & -21.7 & -15.6 & -18.2 & -12.0 & -17.4 & -10.6 & -19.3 & -13.0 \\
\hline $6-311+G(2 d f)$ & -15.0 & -12.5 & -16.9 & -14.3 & -16.5 & -13.8 & -12.1 & -9.5 & -19.3 & -16.5 & -16.9 & -13.0 & -13.0 & -10.5 & -15.6 & -13.1 \\
\hline $6-311 G(3 d f)$ & -17.9 & -12.6 & -20.1 & -14.5 & -19.7 & -14.0 & -14.2 & -9.2 & -22.0 & -16.9 & -18.3 & -12.9 & -16.3 & -10.8 & -18.8 & -13.4 \\
\hline $6-311+G(3 d f)$ & -15.5 & -12.6 & -17.4 & -14.4 & -17.4 & -14.0 & -9.3 & -9.3 & -20.0 & -17.0 & -17.0 & -13.1 & -13.4 & -10.7 & -16.3 & -13.3 \\
\hline cc-pVDZ & -20.8 & -13.8 & -23.0 & -15.8 & -22.8 & -16.5 & -18.0 & -12.2 & -25.6 & -19.6 & -23.5 & -16.9 & -19.7 & -11.6 & -21.2 & -14.2 \\
\hline jul-cc-pVDZ & -15.2 & -11.6 & -17.2 & -13.6 & -18.5 & -14.2 & -14.4 & -10.3 & -21.6 & -17.8 & -20.1 & -15.0 & -12.8 & -9.5 & -16.4 & -12.6 \\
\hline cc-pVTZ & -14.8 & -12.4 & -16.6 & -14.2 & -15.4 & -13.3 & -11.0 & -9.1 & -18.6 & -16.5 & -15.4 & -12.9 & -13.3 & -10.6 & -15.2 & -12.7 \\
\hline jul-cc-pVTZ & -12.3 & -13.2 & -15.1 & -14.1 & -14.4 & -13.5 & -10.3 & -9.2 & -18.2 & -17.1 & -15.3 & -12.8 & -11.3 & -10.3 & -13.7 & -12.8 \\
\hline cc-pVQZ & -13.6 & -12.7 & -15.4 & -14.5 & -14.7 & -13.9 & -10.2 & -9.5 & -17.9 & -17.1 & -14.1 & -12.9 & -11.9 & -10.8 & -14.3 & -13.3 \\
\hline jul-cc-pVQZ & -13.0 & -12.7 & -14.9 & -14.5 & -14.5 & -14.1 & -10.0 & -9.7 & -17.9 & -17.6 & -14.5 & -13.3 & -11.2 & -10.8 & -13.7 & -13.3 \\
\hline Def2-SVP & -24.6 & -15.4 & -27.4 & -17.9 & -28.6 & -19.9 & -21.9 & -13.8 & -30.5 & -22.3 & -28.8 & -19.7 & -23.2 & -12.8 & -25.4 & -16.0 \\
\hline Def2-TZV & -18.6 & -13.7 & -21.5 & -16.4 & -20.6 & -15.2 & -14.9 & -10.0 & -24.6 & -19.2 & -22.9 & -16.8 & -15.9 & -11.0 & -17.6 & -12.6 \\
\hline Def2-TZVP & -14.2 & -12.8 & -15.9 & -14.5 & -14.9 & -13.6 & -10.6 & -9.4 & -18.2 & -16.9 & -15.4 & -13.4 & -12.8 & -11.0 & -14.8 & -13.2 \\
\hline Def2-QzV & -19.0 & -13.6 & -21.9 & -16.4 & -21.4 & -15.5 & -15.4 & -10.0 & -24.8 & -19.2 & -22.2 & -16.1 & -16.2 & -11.0 & -18.4 & -12.8 \\
\hline Def2-QZVP & -13.3 & -13.0 & -15.1 & -14.8 & -14.6 & -14.3 & -10.2 & -9.9 & -17.8 & -17.5 & -13.7 & -13.0 & -11.6 & -11.2 & -14.1 & -13.7 \\
\hline DGDZVP & -17.8 & -14.1 & -20.1 & -16.4 & -20.3 & -16.4 & -15.4 & -11.6 & -24.4 & -20.2 & -20.3 & -16.3 & -15.5 & -11.8 & -18.1 & -14.4 \\
\hline DGDZVP2 & -17.6 & -13.6 & -19.9 & -15.8 & -19.9 & -15.6 & -15.1 & -10.8 & -24.4 & -19.5 & -20.6 & -15.9 & -15.5 & -11.4 & -17.9 & -13.8 \\
\hline DGTZVP & -17.2 & -13.8 & -19.3 & -15.9 & -18.5 & -15.1 & -13.5 & -10.2 & -22.0 & -18.5 & -19.1 & -15.5 & -15.1 & -11.7 & -17.4 & -13.8 \\
\hline LANL2DZ & -21.0 & -15.8 & -24.4 & -19.0 & -25.4 & -19.5 & -20.3 & -14.8 & -29.6 & -23.9 & -29.2 & -22.8 & -18.0 & -12.9 & -20.4 & -15.1 \\
\hline
\end{tabular}


Table S2. CP-corrected and uncorrected interaction energy for Int.1-Br $\left(\mathrm{kJ} \mathrm{mol}^{-1}\right)$ as a function of method and basis set. The benchmark $\mathrm{CCSD}(\mathrm{T}) / \mathrm{CBS}$ value for this interaction is $-21.3 \mathrm{~kJ} \mathrm{~mol}^{-1}$.

\begin{tabular}{|c|c|c|c|c|c|c|c|c|c|c|c|c|c|c|c|c|}
\hline \multirow{2}{*}{$\begin{array}{l}\text { Method } \\
\text { Basis Set }\end{array}$} & \multicolumn{2}{|c|}{ M06-2X } & \multicolumn{2}{|c|}{$\omega B 97 X D$} & \multicolumn{2}{|c|}{ CAM-B3LYP-D3 } & \multicolumn{2}{|c|}{ B3LYP-D3 } & \multicolumn{2}{|c|}{ M11 } & \multicolumn{2}{|c|}{ LC- $\omega$ PBE-D3 } & \multicolumn{2}{|c|}{ BLYP-D3 } & \multicolumn{2}{|c|}{ PBEPBE-D3 } \\
\hline & Uncorr. & Corr. & Uncorr. & Corr. & Uncorr. & Corr. & Uncorr. & Corr. & Uncorr. & Corr. & Uncorr. & Corr. & Uncorr & Corr. & Uncorr & Corr. \\
\hline $6-311 G(d)$ & -26.4 & -19.4 & -11.5 & -18.4 & -25.0 & -17.4 & -22.8 & -15.2 & -23.6 & -16.6 & -24.7 & -17.4 & -20.7 & -12.8 & -22.9 & -15.1 \\
\hline $6-311+G(d)$ & -24.7 & -19.7 & -16.2 & -11.3 & -21.8 & -16.9 & -19.5 & -14.6 & -22.6 & -16.8 & -22.4 & -17.1 & -16.8 & -12.0 & -19.8 & -14.6 \\
\hline $6-311 G(2 d)$ & -21.4 & -16.0 & -14.6 & -9.4 & -20.6 & -14.6 & -18.9 & -13.0 & -17.9 & -12.6 & -19.8 & -14.4 & -17.4 & -11.1 & -19.4 & -13.5 \\
\hline $6-311+G(2 d)$ & -19.5 & -16.7 & -12.4 & -9.7 & -17.2 & -14.6 & -15.5 & -12.9 & -16.8 & -13.3 & -17.4 & -14.5 & -13.4 & -10.9 & -16.1 & -13.4 \\
\hline 6-311G(df) & -26.2 & -19.0 & -18.3 & -11.2 & -25.0 & -17.1 & -22.7 & -15.0 & -23.2 & -15.9 & -24.5 & -17.0 & -20.7 & -12.5 & -22.8 & -14.9 \\
\hline $6-311+G(d f)$ & -24.5 & -19.3 & -16.2 & -11.1 & -21.7 & -16.7 & -19.4 & -14.4 & -22.3 & -16.3 & -22.1 & -16.7 & -16.7 & -11.8 & -19.6 & -14.4 \\
\hline $6-311 G(2 d f)$ & -21.4 & -16.1 & -14.6 & -9.5 & -20.7 & -14.7 & -18.9 & -13.1 & -17.9 & -12.6 & -19.8 & -14.3 & -17.3 & -11.1 & -19.2 & -13.4 \\
\hline $6-311+G(2 d f)$ & -19.4 & -16.8 & -12.3 & -9.8 & -17.1 & -14.7 & -15.4 & -13.0 & -16.9 & -13.3 & -17.1 & -14.5 & -13.2 & -10.9 & -15.8 & -13.3 \\
\hline $6-311 G(3 d f)$ & -22.0 & -17.2 & -14.4 & -9.6 & -20.3 & -14.9 & -13.2 & -18.2 & -18.5 & -13.3 & -20.1 & -14.7 & -16.4 & -11.2 & -18.9 & -13.7 \\
\hline $6-311+G(3 d f)$ & -20.0 & -17.2 & -12.5 & -9.7 & -17.5 & -14.9 & -15.7 & -13.1 & -17.0 & -13.4 & -17.8 & -14.7 & -13.5 & -11.0 & -16.3 & -13.6 \\
\hline cc-pVDZ & -26.2 & -20.2 & -18.7 & -12.8 & -23.5 & -16.3 & -21.4 & -14.4 & -24.3 & -17.7 & -23.8 & -17.4 & -20.2 & -12.0 & -21.6 & -14.6 \\
\hline jul-cc-pVDZ & -22.1 & -18.2 & -14.9 & -10.7 & -17.6 & -14.0 & -15.7 & -12.0 & -20.8 & -15.5 & -19.3 & -15.0 & -13.2 & -9.7 & -16.7 & -12.8 \\
\hline cc-pVTZ & -18.8 & -16.8 & -11.3 & -9.4 & -16.8 & -14.5 & -15.1 & -12.8 & -15.8 & -13.3 & -15.9 & -13.9 & -13.5 & -10.8 & -15.3 & -12.9 \\
\hline jul-cc-pVTZ & -18.6 & -17.4 & -10.7 & -9.6 & -15.4 & -14.5 & -13.7 & -12.8 & -16.1 & -13.2 & -15.2 & -14.1 & -11.6 & -10.6 & -14.0 & -13.0 \\
\hline cc-pVQZ & -18.3 & -17.5 & -10.7 & -9.9 & -15.7 & -14.9 & -14.0 & -13.1 & -14.7 & -13.4 & -15.4 & -14.6 & -12.2 & -11.1 & -14.6 & -13.5 \\
\hline jul-cc-pVQZ & -18.4 & -18.0 & -10.5 & -10.1 & -15.3 & -14.9 & -13.5 & -13.1 & -15.1 & -13.7 & -15.3 & -14.9 & -11.5 & -11.1 & -14.0 & -13.6 \\
\hline Def2-SVP & -31.3 & -23.3 & -22.6 & -14.9 & -27.8 & -18.7 & -25.0 & -16.1 & -29.7 & -21.0 & -29.5 & -21.2 & -23.4 & -5.6 & -16.7 & -25.7 \\
\hline Def2-TZV & -24.6 & -19.4 & -15.1 & -10.2 & -21.7 & -16.7 & -18.8 & -14.1 & -23.4 & -17.2 & -21.1 & -15.8 & -15.9 & -3.8 & -12.6 & -17.5 \\
\hline Def2-TZVP & -18.4 & -17.2 & -11.0 & -9.7 & -16.3 & -14.8 & -14.6 & -13.2 & -15.9 & -13.8 & -15.6 & -14.2 & -13.0 & -3.1 & -13.3 & -14.9 \\
\hline Def2-QZV & -24.2 & -19.1 & -15.1 & -10.1 & -21.4 & -16.3 & -18.7 & -13.8 & -21.9 & -16.1 & -21.2 & -15.8 & -15.8 & -3.8 & -12.6 & -17.9 \\
\hline Def2-QZVP & -18.2 & -17.8 & -10.7 & -10.3 & -15.5 & -15.1 & -13.8 & -13.4 & -14.2 & -13.4 & -15.4 & -15.0 & -11.9 & -2.9 & -13.9 & -14.4 \\
\hline DGDZVP & -24.6 & -20.5 & -15.8 & -12.0 & -20.7 & -16.9 & -18.5 & -14.8 & -20.6 & -4.9 & -21.0 & -17.2 & -16.1 & -14.7 & -18.5 & -18.5 \\
\hline LANL2DZ & -30.3 & -24.5 & -21.2 & -15.4 & -25.0 & -19.5 & -21.6 & -16.3 & -30.1 & -7.2 & -26.7 & -20.5 & -18.4 & -15.5 & -20.9 & -20.9 \\
\hline
\end{tabular}


Table S3. CP-corrected and uncorrected interaction energy for Int.1-F $\left(\mathrm{kJ} \mathrm{mol}^{-1}\right)$ as a function of method and basis set. The benchmark $\mathrm{CCSD}(\mathrm{T}) / \mathrm{CBS}$ value for this interaction is $-19.1 \mathrm{~kJ} \mathrm{~mol}^{-1}$.

\begin{tabular}{|c|c|c|c|c|c|c|c|c|c|c|c|c|c|c|c|c|}
\hline \multirow{2}{*}{$\begin{array}{l}\text { Method } \\
\text { Basis Set }\end{array}$} & \multicolumn{2}{|c|}{ M06-2X } & \multicolumn{2}{|c|}{$\omega B 97 X D$} & \multicolumn{2}{|c|}{ CAM-B3LYP-D3 } & \multicolumn{2}{|c|}{ B3LYP-D3 } & \multicolumn{2}{|c|}{ M11 } & \multicolumn{2}{|c|}{ LC- $\omega$ PBE-D3 } & \multicolumn{2}{|c|}{ BLYP-D3 } & \multicolumn{2}{|c|}{ PBEPBE-D3 } \\
\hline & Uncorr. & Corr. & Uncorr. & Corr. & Uncorr. & Corr. & Uncorr. & Corr. & Uncorr. & Corr. & Uncorr. & Corr. & Uncorr & Corr. & Uncorr & Corr. \\
\hline $6-311 G(d)$ & -26.2 & -19.0 & -18.5 & -11.4 & -24.4 & -16.4 & -21.9 & -14.0 & -23.1 & -15.8 & -23.4 & -15.7 & -20.4 & -11.8 & -22.9 & -14.7 \\
\hline $6-311+G(d)$ & -24.0 & -18.8 & -16.1 & -10.9 & -20.6 & -15.6 & -18.2 & -13.2 & -21.0 & -15.4 & -20.5 & -15.0 & -15.9 & -11.0 & -19.4 & -14.1 \\
\hline $6-311 G(2 d)$ & -21.4 & -15.3 & -14.9 & -9.1 & -20.5 & -13.6 & -18.3 & -11.7 & -17.8 & -11.6 & -18.8 & -12.4 & -17.4 & -10.1 & -19.7 & -12.8 \\
\hline $6-311+G(2 d)$ & -18.5 & -15.9 & -11.9 & -9.2 & -15.9 & -13.3 & -14.0 & -11.5 & -15.3 & -12.0 & -15.1 & -12.4 & -12.3 & -9.8 & -15.4 & -12.8 \\
\hline cc-pVDZ & -24.8 & -18.8 & -17.9 & -12.0 & -22.1 & -15.0 & -19.8 & -12.9 & -22.3 & -15.7 & -21.5 & -15.0 & -19.0 & -11.0 & -20.8 & -13.8 \\
\hline jul-cc-pVDZ & -20.4 & -16.9 & -13.8 & -10.0 & -16.3 & -12.8 & -14.2 & -10.8 & -18.2 & -13.6 & -16.7 & -12.6 & -12.1 & -8.8 & -15.8 & -12.2 \\
\hline cc-pVTZ & -18.4 & -16.2 & -11.3 & -9.2 & -16.1 & -13.5 & -14.1 & -11.5 & -15.1 & -12.4 & -14.4 & -12.1 & -12.8 & -9.9 & -15.1 & -12.4 \\
\hline jul-cc-pVTZ & -17.7 & -16.6 & -10.3 & -9.3 & -14.3 & -13.4 & -12.3 & -11.4 & -14.4 & -12.1 & -13.2 & -12.3 & -10.5 & -9.6 & -13.3 & -12.4 \\
\hline Def2-SVP & -29.0 & -21.8 & -21.3 & -14.1 & -25.5 & -17.2 & -22.6 & -14.6 & -27.0 & -18.9 & -26.4 & -18.6 & -21.2 & -12.1 & -24.0 & -15.8 \\
\hline Def2-TZV & -24.3 & -19.1 & -15.3 & -10.4 & -21.0 & -16.0 & -18.0 & -13.1 & -14.9 & -12.8 & -19.6 & -14.4 & -15.4 & -10.6 & -17.6 & -12.6 \\
\hline Def2-TZVP & -17.6 & -16.4 & -10.6 & -9.5 & -15.1 & -13.7 & -13.2 & -11.8 & -14.9 & -12.8 & -13.6 & -12.3 & -11.9 & -10.2 & -14.4 & -12.8 \\
\hline Def2-QZV & -23.9 & -18.6 & -15.2 & -10.1 & -20.7 & -15.5 & -17.8 & -12.8 & -21.0 & -15.2 & -19.7 & -14.2 & -15.2 & -10.2 & -17.7 & -12.5 \\
\hline DGDZVP & -23.8 & -20.1 & -15.4 & -11.9 & -19.2 & -15.8 & -16.8 & -13.4 & -19.7 & -16.1 & -19.0 & -15.4 & -14.7 & -11.2 & -17.7 & -14.2 \\
\hline LANL2DZ & -29.6 & -23.7 & -21.1 & -15.2 & -24.3 & -18.8 & -20.8 & -15.5 & -29.0 & -22.3 & -24.9 & -18.7 & -17.8 & -12.6 & -20.8 & -15.3 \\
\hline
\end{tabular}


Table S4. CP-corrected and uncorrected interaction energy for Int.2-Cl $\left(\mathrm{kJ} \mathrm{mol}^{-1}\right)$ as a function of method and basis set. The benchmark $\operatorname{CCSD}(\mathrm{T}) / \mathrm{CBS}$ value for this interaction is $-19.7 \mathrm{~kJ} \mathrm{~mol}^{-1}$.

\begin{tabular}{|c|c|c|c|c|c|c|c|c|c|c|c|c|c|c|c|c|}
\hline \multirow{2}{*}{$\begin{array}{l}\text { Method } \\
\text { Basis Set }\end{array}$} & \multicolumn{2}{|c|}{ M06-2X } & \multicolumn{2}{|c|}{$\omega B 97 X D$} & \multicolumn{2}{|c|}{ CAM-B3LYP-D3 } & \multicolumn{2}{|c|}{ B3LYP-D3 } & \multicolumn{2}{|c|}{ M11 } & \multicolumn{2}{|c|}{ LC- $\omega$ PBE-D3 } & \multicolumn{2}{|c|}{ BLYP-D3 } & \multicolumn{2}{|c|}{ PBEPBE-D3 } \\
\hline & Uncorr. & Corr. & Uncorr. & Corr. & Uncorr. & Corr. & Uncorr. & Corr. & Uncorr. & Corr. & Uncorr. & Corr. & Uncorr & Corr. & Uncorr & Corr. \\
\hline $6-311 G(d)$ & -25.1 & -15.9 & -19.0 & -10.3 & -24.2 & -15.3 & -25.2 & -16.5 & -25.0 & -14.8 & -25.3 & -16.4 & -25.9 & -17.4 & -20.8 & -12.4 \\
\hline $6-311+G(d)$ & -25.7 & -17.2 & -19.1 & -10.8 & -23.3 & -15.6 & -24.1 & -16.5 & -26.9 & -16.0 & -25.3 & -16.9 & -24.6 & -17.3 & -20.3 & -12.5 \\
\hline $6-311 G(2 d)$ & -22.1 & -14.3 & -16.7 & -9.4 & -22.4 & -14.8 & -23.7 & -16.3 & -21.1 & -12.3 & -22.7 & -15.3 & -24.9 & -17.5 & -19.2 & -12.2 \\
\hline $6-311+G(2 d)$ & -21.0 & -15.6 & -15.2 & -9.9 & -20.1 & -15.1 & -21.3 & -16.4 & -21.7 & -13.7 & -21.1 & -15.8 & -22.1 & -17.5 & -17.1 & -12.3 \\
\hline $6-311 G(d f)$ & -26.0 & -16.4 & -19.8 & -10.8 & -24.8 & -15.4 & -25.8 & -16.7 & -25.9 & -15.2 & -26.1 & -16.8 & -26.4 & -17.5 & -21.3 & -12.7 \\
\hline $6-311+G(d f)$ & -26.5 & -18.0 & -19.6 & -11.4 & -23.5 & -15.8 & -24.3 & -16.7 & -27.7 & -16.7 & -25.7 & -17.4 & -24.7 & -17.5 & -20.5 & -12.9 \\
\hline $6-311 G(2 d f)$ & -23.3 & -15.0 & -17.6 & -9.9 & -23.0 & -14.9 & -24.2 & -16.4 & -27.7 & -16.7 & -23.5 & -15.7 & -25.3 & -17.6 & -19.7 & -12.4 \\
\hline $6-311+G(2 d f)$ & -21.8 & -16.4 & -15.6 & -10.5 & -20.1 & -15.4 & -21.3 & -16.6 & -22.5 & -14.5 & -21.4 & -16.3 & -22.1 & -17.6 & -17.2 & -12.6 \\
\hline $6-311 G(3 d f)$ & -24.4 & -16.9 & -17.8 & -10.5 & -22.9 & -15.4 & -23.9 & -16.7 & -23.6 & -14.6 & -24.2 & -16.7 & -25.0 & -17.7 & -19.9 & -12.8 \\
\hline $6-311+G(3 d f)$ & -23.0 & -17.4 & -16.2 & -10.7 & -20.5 & -15.5 & -21.5 & -16.5 & -23.8 & -15.2 & -22.5 & -16.9 & -22.2 & -17.5 & -17.7 & -12.8 \\
\hline cc-pVDZ & -22.8 & -17.1 & -16.7 & -11.3 & -21.3 & -15.0 & -21.9 & -15.8 & -23.4 & -17.3 & -23.8 & -18.2 & -23.6 & -16.7 & -17.9 & -11.7 \\
\hline jul-cc-pVDZ & -22.5 & -19.0 & -15.8 & -12.0 & -18.5 & -15.4 & -19.2 & -16.1 & -23.5 & -17.9 & -22.5 & -18.2 & -19.7 & -16.9 & -16.2 & -12.7 \\
\hline cc-pVTZ & -15.9 & -18.5 & -12.8 & -10.7 & -18.3 & -15.5 & -19.5 & -16.8 & -17.8 & -14.3 & -19.0 & -16.4 & -21.0 & -18.0 & -15.4 & -12.6 \\
\hline jul-cc-pVTZ & -18.3 & -16.8 & -12.0 & -10.8 & -16.7 & -15.6 & -17.9 & -16.9 & -18.5 & -14.2 & -17.9 & -16.7 & -19.0 & -18.0 & -13.9 & -13.0 \\
\hline cc-pVQZ & -16.9 & -15.8 & -12.8 & -10.7 & -16.8 & -15.6 & -18.2 & -17.0 & -14.8 & -13.1 & -17.5 & -16.4 & -19.7 & -18.2 & -14.3 & -12.9 \\
\hline jul-cc-pVQZ & -17.0 & -16.6 & -10.9 & -10.5 & -16.1 & -15.7 & -17.4 & -17.1 & -15.0 & -13.4 & -17.1 & -16.7 & -18.7 & -18.3 & -13.6 & -13.1 \\
\hline Def2-SVP & -26.9 & -16.0 & -18.9 & -8.6 & -26.9 & -14.6 & -27.1 & -15.0 & -27.9 & -16.4 & -27.9 & -17.4 & -29.5 & -16.0 & -22.1 & -10.1 \\
\hline Def2-TZV & -24.8 & -17.0 & -18.3 & -10.9 & -24.4 & -16.7 & -24.8 & -17.4 & -23.6 & -15.8 & -25.8 & -17.7 & -25.5 & -18.1 & -20.3 & -12.6 \\
\hline Def2-TZVP & -17.6 & -15.6 & -11.9 & -10.1 & -17.6 & -15.4 & -18.9 & -16.8 & -16.7 & -13.8 & -18.1 & -16.1 & -20.6 & -18.0 & -14.9 & -12.6 \\
\hline Def2-QZV & -21.4 & -15.3 & -15.1 & -9.3 & -21.8 & -15.9 & -22.3 & -16.7 & -20.2 & -13.3 & -23.0 & -16.7 & -22.9 & -17.4 & -17.8 & -11.8 \\
\hline Def2-QZVP & -16.6 & -16.1 & -10.8 & -10.3 & -16.2 & -15.6 & -17.5 & -16.9 & -13.8 & -12.7 & -17.1 & -16.5 & -18.9 & -18.2 & -13.7 & -13.0 \\
\hline DGDZVP & -23.1 & -18.2 & -16.4 & -12.0 & -19.9 & -15.9 & -20.8 & -16.9 & -21.3 & -16.4 & -22.6 & -17.9 & -21.3 & -17.7 & -16.9 & -12.8 \\
\hline DGDZVP2 & -22.8 & -17.4 & -16.5 & -11.5 & -20.4 & -15.9 & -21.4 & -17.0 & -20.7 & -15.5 & -22.6 & -17.4 & -22.0 & -17.8 & -17.3 & -12.7 \\
\hline DGTZVP & -19.4 & -14.6 & -14.2 & -9.6 & -20.1 & -15.4 & -21.3 & -16.7 & -18.7 & -13.7 & -20.8 & -15.9 & -22.3 & -17.7 & -16.9 & -12.1 \\
\hline LANL2DZ & -28.9 & -21.3 & -20.3 & -13.1 & -24.1 & -16.5 & -23.6 & -16.2 & -29.9 & -21.9 & -28.5 & -20.9 & -24.0 & -16.2 & -19.6 & -11.9 \\
\hline
\end{tabular}


Table S5. CP-corrected and uncorrected interaction energy for Int.2-Br $\left(\mathrm{kJ} \mathrm{mol}^{-1}\right)$ as a function of method and basis set. The benchmark $\mathrm{CCSD}(\mathrm{T}) / \mathrm{CBS}$ value for this interaction is $-21.3 \mathrm{~kJ} \mathrm{~mol}^{-1}$.

\begin{tabular}{|c|c|c|c|c|c|c|c|c|c|c|c|c|c|c|c|c|}
\hline \multirow{2}{*}{$\begin{array}{l}\text { Method } \\
\text { Basis Set }\end{array}$} & \multicolumn{2}{|c|}{ M06-2X } & \multicolumn{2}{|c|}{$\omega B 97 X D$} & \multicolumn{2}{|c|}{ CAM-B3LYP-D3 } & \multicolumn{2}{|c|}{ B3LYP-D3 } & \multicolumn{2}{|c|}{ M11 } & \multicolumn{2}{|c|}{ LC- $\omega$ PBE-D3 } & \multicolumn{2}{|c|}{ BLYP-D3 } & \multicolumn{2}{|c|}{ PBEPBE-D3 } \\
\hline & Uncorr. & Corr. & Uncorr. & Corr. & Uncorr. & Corr. & Uncorr. & Corr. & Uncorr. & Corr. & Uncorr. & Corr. & Uncorr. & Corr. & Uncorr. & Corr. \\
\hline $6-311 G(d)$ & -21.4 & -15.1 & -16.3 & -10.3 & -22.7 & -16.2 & -24.4 & -18.0 & -19.9 & -13.2 & -24.4 & -18.2 & -25.2 & -18.6 & -18.2 & -11.7 \\
\hline $6-311+G(d)$ & -22.7 & -16.9 & -16.9 & -11.4 & -22.3 & -16.9 & -24.0 & -18.7 & -21.8 & -14.4 & -24.8 & -19.0 & -24.4 & -19.2 & -18.4 & -12.7 \\
\hline $6-311 G(2 d)$ & -20.8 & -14.6 & -16.1 & -10.1 & -22.9 & -16.3 & -24.9 & -18.4 & -18.2 & -11.7 & -23.8 & -17.7 & -26.1 & -19.3 & -18.6 & -12.2 \\
\hline $6-311+G(2 d)$ & -20.5 & -16.0 & -15.6 & -11.0 & -21.4 & -16.9 & -23.4 & -18.9 & -18.7 & -12.7 & -23.2 & -18.4 & -24.0 & -19.7 & -17.4 & -13.0 \\
\hline $6-311 G(d f)$ & -22.2 & -15.8 & -17.0 & -10.9 & -23.2 & -16.5 & -24.9 & -18.3 & -20.6 & -13.8 & -25.0 & -18.7 & -25.7 & -18.9 & -18.7 & -12.2 \\
\hline $6-311+G(d f)$ & -23.4 & -17.7 & -17.4 & -12.0 & -22.4 & -17.2 & -24.1 & -19.0 & -22.5 & -15.1 & -25.2 & -19.5 & -24.5 & -19.5 & -18.6 & -13.1 \\
\hline $6-311 G(2 d f)$ & -21.6 & -15.3 & -16.7 & -10.7 & -23.2 & -16.6 & -25.2 & -18.7 & -19.0 & -12.3 & -24.4 & -18.3 & -26.3 & -19.5 & -19.0 & -12.6 \\
\hline $6-311+G(2 d f)$ & -21.0 & -16.8 & -16.0 & -11.6 & -21.3 & -17.2 & -23.3 & -19.1 & -19.4 & -13.5 & -23.4 & -19.0 & -23.9 & -19.9 & -17.4 & -13.3 \\
\hline $6-311 G(3 d f)$ & -22.3 & -17.4 & -16.6 & -11.8 & -22.6 & -17.3 & -24.4 & -19.2 & -20.2 & -14.3 & -24.5 & -19.5 & -20.0 & -25.5 & -18.7 & -13.4 \\
\hline $6-311+G(3 d f)$ & -21.0 & -17.9 & -15.1 & -12.0 & -20.1 & -17.3 & -21.8 & -19.1 & -20.3 & -14.8 & -22.9 & -19.7 & -22.3 & -19.8 & -16.2 & -13.5 \\
\hline cc-pVDZ & -23.6 & -18.3 & -17.6 & -12.6 & -22.5 & -16.4 & -23.8 & -17.9 & -23.1 & -17.4 & -26.3 & -21.1 & -25.0 & -18.2 & -18.0 & -12.1 \\
\hline jul-cc-pVDZ & -24.7 & -20.3 & -17.6 & -13.6 & -20.5 & -17.2 & -21.9 & -18.5 & -24.3 & -18.3 & -25.9 & -21.4 & -22.0 & -18.9 & -17.3 & -13.5 \\
\hline cc-pVTZ & -18.5 & -16.1 & -13.5 & -11.3 & -19.4 & -17.0 & -21.3 & -19.0 & -17.0 & -13.6 & -21.2 & -18.9 & -22.3 & -19.7 & -15.3 & -12.9 \\
\hline jul-cc-pVTZ & -19.2 & -17.6 & -13.3 & -12.0 & -18.5 & -17.4 & -20.3 & -19.2 & -19.7 & -14.4 & -20.8 & -19.5 & -21.1 & -20.0 & -14.6 & -13.5 \\
\hline cc-pVQZ & -18.0 & -16.9 & -12.6 & -11.6 & -18.5 & -17.3 & -20.4 & -19.2 & -15.0 & -13.1 & -20.4 & -19.3 & -21.4 & -20.0 & -14.7 & -13.4 \\
\hline jul-cc-pVQZ & -18.2 & -17.7 & -12.5 & -12.0 & -17.9 & -17.4 & -19.8 & -19.4 & -14.9 & -13.2 & -20.1 & -19.6 & -20.8 & -20.2 & -14.2 & -13.7 \\
\hline Def2-SVP & -26.8 & -19.0 & -19.1 & -11.9 & -25.4 & -16.6 & -26.4 & -17.7 & -26.3 & -18.3 & -28.9 & -21.7 & -28.0 & -18.0 & -20.5 & -11.8 \\
\hline Def2-TZV & -24.1 & -16.7 & -18.2 & -11.0 & -24.6 & -17.3 & -25.6 & -18.7 & -22.2 & -14.7 & -27.1 & -19.2 & -25.9 & -18.9 & -19.4 & -12.0 \\
\hline Def2-TZVP & -18.2 & -16.4 & -13.1 & -11.3 & -19.1 & -17.1 & -21.0 & -19.0 & -16.6 & -13.6 & -20.8 & -18.9 & -22.0 & -19.8 & -15.1 & -13.0 \\
\hline Def2-QZV & -20.4 & -15.7 & -14.9 & -10.5 & -21.8 & -17.2 & -23.1 & -18.7 & -17.7 & -12.2 & -23.8 & -18.8 & -23.6 & -19.3 & -17.0 & -12.3 \\
\hline Def2-QZVP & -17.6 & -17.0 & -12.3 & -11.7 & -18.0 & -17.3 & -19.9 & -19.2 & -13.9 & -12.6 & -20.1 & -19.4 & -20.9 & -20.1 & -14.4 & -13.5 \\
\hline DGDZVP & -21.6 & -16.8 & -16.0 & -11.5 & -20.9 & -16.8 & -22.6 & -18.6 & -18.1 & -13.4 & -23.7 & -19.1 & -23.0 & -19.2 & -16.6 & -12.4 \\
\hline LANL2DZ & -28.6 & -21.1 & -20.9 & -13.8 & -24.2 & -17.0 & -24.5 & -17.5 & -27.8 & -19.9 & -30.4 & -22.8 & -24.3 & -17.2 & -19.1 & -11.9 \\
\hline
\end{tabular}


Table S6. CP-corrected and uncorrected interaction energy for Int.2-F $\left(\mathrm{kJ} \mathrm{mol}^{-1}\right)$ as a function of method and basis set. The benchmark $\mathrm{CCSD}(\mathrm{T}) / \mathrm{CBS}$ value for this interaction is $-12.1 \mathrm{~kJ} \mathrm{~mol}^{-1}$.

\begin{tabular}{|c|c|c|c|c|c|c|c|c|c|c|c|c|c|c|c|c|}
\hline \multirow{2}{*}{$\begin{array}{l}\text { Method } \\
\text { Basis Set }\end{array}$} & \multicolumn{2}{|c|}{ M06-2X } & \multicolumn{2}{|c|}{$\omega B 97 X D$} & \multicolumn{2}{|c|}{ CAM-B3LYP-D3 } & \multicolumn{2}{|c|}{ B3LYP-D3 } & \multicolumn{2}{|c|}{ M11 } & \multicolumn{2}{|c|}{ LC- $\omega$ PBE-D3 } & \multicolumn{2}{|c|}{ BLYP-D3 } & \multicolumn{2}{|c|}{ PBEPBE-D3 } \\
\hline & Uncorr. & Corr. & Uncorr. & Corr. & Uncorr. & Corr. & Uncorr. & Corr. & Uncorr. & Corr. & Uncorr. & Corr. & Uncorr & Corr. & Uncorr & Corr. \\
\hline $6-311 G(d)$ & -15.7 & -8.5 & -15.8 & -8.7 & -18.7 & -10.5 & -19.3 & -11.3 & -15.8 & -8.2 & -17.4 & -9.6 & -20.2 & -11.4 & -18.8 & -10.8 \\
\hline $6-311+G(d)$ & -15.0 & -9.2 & -15.2 & -9.5 & -16.5 & -11.2 & -17.0 & -11.7 & -15.5 & -8.9 & -16.3 & -10.5 & -16.5 & -11.5 & -17.1 & -11.5 \\
\hline $6-311 G(2 d)$ & -15.2 & -7.2 & -15.6 & -7.8 & -18.8 & -9.7 & -19.6 & -10.8 & -14.7 & -6.4 & -17.3 & -8.7 & -20.8 & -11.0 & -19.4 & -10.3 \\
\hline $6-311+G(2 d)$ & -11.4 & -7.7 & -12.1 & -8.4 & -13.6 & -10.1 & -14.3 & -10.8 & -11.5 & -6.8 & -13.0 & -9.2 & -14.1 & -10.8 & -14.3 & -10.7 \\
\hline cc-pVDZ & -11.9 & -6.9 & -12.6 & -7.7 & -15.2 & -9.5 & -16.2 & -10.7 & -11.7 & -6.2 & -14.1 & -8.7 & -17.2 & -11.1 & -15.3 & -9.7 \\
\hline jul-cc-pVDZ & -10.2 & -7.9 & -11.0 & -8.5 & -12.4 & -10.1 & -13.0 & -10.8 & -10.0 & -7.2 & -12.1 & -9.4 & -12.8 & -10.6 & -11.8 & -10.8 \\
\hline cc-pVTZ & -11.5 & -8.2 & -11.9 & -8.6 & -13.8 & -9.9 & -14.2 & -10.5 & -12.2 & -8.4 & -13.1 & -9.4 & -14.8 & -10.5 & -14.2 & -10.3 \\
\hline jul-cc-pVTZ & -10.0 & -8.5 & -10.3 & -8.9 & -11.2 & -10.2 & -11.8 & -10.8 & -10.9 & -7.6 & -10.9 & -9.7 & -11.6 & -10.6 & -11.8 & -10.8 \\
\hline Def2-SVP & -12.8 & -7.4 & -13.6 & -8.3 & -16.2 & -10.2 & -17.0 & -11.2 & -12.5 & -6.6 & -15.0 & -9.3 & -18.1 & -11.6 & -15.9 & -10.0 \\
\hline Def2-TZV & -17.0 & -11.5 & -16.9 & -11.8 & -18.8 & -13.4 & -18.8 & -13.7 & -18.5 & -12.5 & -18.9 & -13.0 & -18.4 & -13.1 & -18.6 & -13.1 \\
\hline Def2-QZV & -13.9 & -10.4 & -14.4 & -11.1 & -16.7 & -13.3 & -17.1 & -13.9 & -13.5 & -9.2 & -16.2 & -12.6 & -16.9 & -13.6 & -16.8 & -13.3 \\
\hline DGDZVP & -13.3 & -10.3 & -12.8 & -9.8 & -13.6 & -10.6 & -13.7 & -10.8 & -14.3 & -11.2 & -10.5 & -13.7 & -13.6 & -10.5 & -13.7 & -10.6 \\
\hline LANL2DZ & -15.6 & -12.0 & -14.8 & -11.2 & -16.6 & -12.7 & -16.6 & -12.7 & -16.7 & -12.8 & -16.4 & -12.4 & -16.4 & -12.3 & -15.9 & -11.9 \\
\hline
\end{tabular}


Table S7. CP-corrected and uncorrected interaction energy for Int.3-Cl $\left(\mathrm{kJ} \mathrm{mol}^{-1}\right)$ as a function of method and basis set. The benchmark $\operatorname{CCSD}(\mathrm{T}) / \mathrm{CBS}$ value for this interaction is $-16.3 \mathrm{~kJ} \mathrm{~mol}^{-1}$.

\begin{tabular}{|c|c|c|c|c|c|c|c|c|c|c|c|c|c|c|c|c|}
\hline \multirow{2}{*}{$\begin{array}{l}\text { Method } \\
\text { Basis Set }\end{array}$} & \multicolumn{2}{|c|}{ M06-2X } & \multicolumn{2}{|c|}{$\omega B 97 X D$} & \multicolumn{2}{|c|}{ CAM-B3LYP-D3 } & \multicolumn{2}{|c|}{ B3LYP-D3 } & \multicolumn{2}{|c|}{ M11 } & \multicolumn{2}{|c|}{ LC- $\omega$ PBE-D3 } & \multicolumn{2}{|c|}{ BLYP-D3 } & \multicolumn{2}{|c|}{ PBEPBE-D3 } \\
\hline & Uncorr. & Corr. & Uncorr. & Corr. & Uncorr. & Corr. & Uncorr. & Corr. & Uncorr. & Corr. & Uncorr. & Corr. & Uncorr & Corr. & Uncorr & Corr. \\
\hline $6-311 G(d)$ & -22.9 & -16.1 & -16.9 & -10.2 & -22.2 & -14.8 & -20.3 & -13.0 & -20.7 & -13.6 & -21.8 & -14.8 & -18.5 & -10.8 & -18.8 & -11.5 \\
\hline $6-311+G(d)$ & -22.3 & -16.0 & -16.2 & -10.2 & -20.7 & -15.0 & -18.8 & -13.1 & -20.8 & -13.4 & -21.1 & -14.8 & -16.4 & -11.0 & -17.4 & -11.6 \\
\hline $6-311 G(2 d)$ & -19.8 & -13.2 & -15.1 & -8.7 & -19.8 & -12.6 & -18.3 & -11.3 & -17.5 & -10.7 & -19.2 & -12.5 & -16.9 & -9.5 & -17.1 & -10.2 \\
\hline $6-311+G(2 d)$ & -16.4 & -13.4 & -12.0 & -9.0 & -12.9 & -15.7 & -14.3 & -11.6 & -15.1 & -10.8 & -15.7 & -12.7 & -12.3 & -9.8 & -13.0 & -10.4 \\
\hline cc-pVDZ & -21.3 & -14.5 & -16.1 & -9.4 & -21.2 & -13.0 & -19.6 & -11.7 & -19.4 & -12.0 & -20.8 & -13.6 & -18.8 & -9.7 & -18.0 & -10.0 \\
\hline jul-cc-pVDZ & -16.5 & -13.8 & -12.0 & -9.2 & -15.0 & -12.5 & -13.8 & -11.3 & -15.2 & -11.3 & -15.7 & -12.5 & -11.8 & -9.4 & -12.7 & -10.1 \\
\hline cc-pVTZ & -15.4 & -13.1 & -10.8 & -8.5 & -15.5 & -12.7 & -14.3 & -11.5 & -13.4 & -10.4 & -14.4 & -11.9 & -13.1 & -9.9 & -12.8 & -9.9 \\
\hline jul-cc-pVTZ & -13.9 & -12.9 & -9.2 & -8.3 & -13.3 & -12.6 & -12.3 & -11.5 & -11.6 & -9.3 & -12.5 & -11.7 & -10.7 & -9.9 & -10.8 & -10.0 \\
\hline Def2-SVP & -22.6 & -15.4 & -17.1 & -9.9 & -22.6 & -13.8 & -20.8 & -12.3 & -20.5 & -12.8 & -22.6 & -15.0 & -19.8 & -10.0 & -19.0 & -10.5 \\
\hline Def2-TZV & -20.8 & -14.7 & -14.4 & -8.7 & -20.1 & -14.5 & -18.2 & -12.8 & -20.2 & -13.3 & -19.1 & -13.1 & -15.8 & -10.5 & -15.6 & -10.1 \\
\hline Def2-TZVP & -14.2 & -12.9 & -9.5 & -8.2 & -14.1 & -12.6 & -13.0 & -11.5 & -12.7 & -10.5 & -13.1 & -11.8 & -11.7 & -9.8 & -11.5 & -9.8 \\
\hline Def2-QZV & -20.5 & -13.6 & -14.4 & -7.9 & -20.6 & -14.1 & -18.8 & -12.6 & -17.9 & -10.6 & -19.6 & -12.6 & -16.5 & -10.4 & -16.5 & -10.1 \\
\hline DGDZVP & -21.6 & -17.6 & -15.1 & -11.5 & -18.2 & -15.0 & -16.5 & -13.4 & -18.1 & -14.1 & -18.8 & -15.2 & -14.2 & -11.1 & -15.1 & -11.9 \\
\hline DGTZVP & -18.1 & -14.7 & -12.3 & -9.1 & -17.2 & -14.1 & -15.6 & -12.6 & -16.4 & -12.6 & -16.4 & -13.3 & -13.7 & -10.7 & -13.9 & -10.8 \\
\hline LANL2DZ & -24.2 & -18.0 & -17.7 & -11.5 & -21.6 & -15.5 & -19.2 & -13.4 & -23.1 & -16.0 & -22.2 & -15.6 & -16.6 & -10.6 & -16.8 & -10.8 \\
\hline
\end{tabular}


Table S8. CP-corrected and uncorrected interaction energy for Int.3-Br $\left(\mathrm{kJ} \mathrm{mol}^{-1}\right)$ as a function of method and basis set. The benchmark $\operatorname{CCSD}(\mathrm{T}) / \mathrm{CBS}$ value for this interaction is $-17.8 \mathrm{~kJ} \mathrm{~mol}^{-1}$.

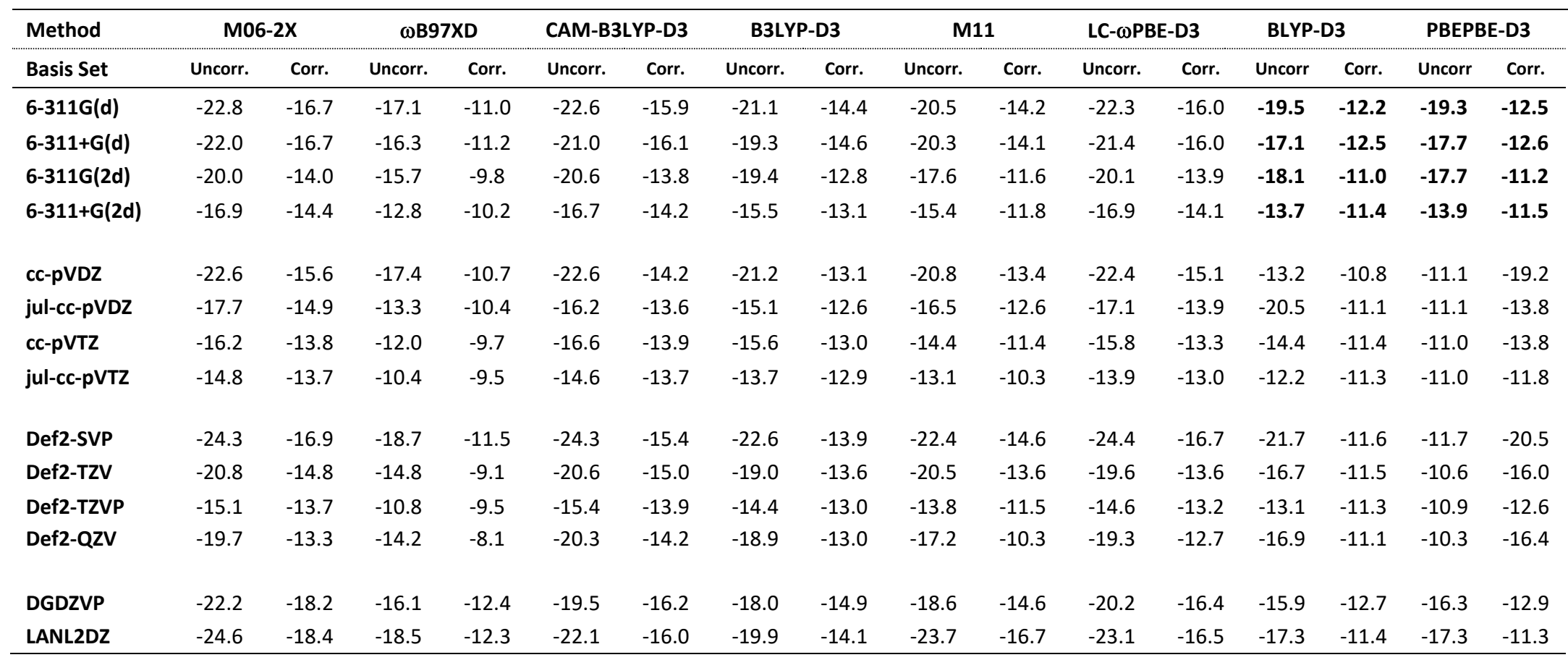


Table S9. CP-corrected and uncorrected interaction energy for Int.3-F $\left(\mathrm{kJ} \mathrm{mol}^{-1}\right)$ as a function of method and basis set. The benchmark $\mathrm{CCSD}(\mathrm{T}) / \mathrm{CBS}$ value for this interaction is $-19.8 \mathrm{~kJ} \mathrm{~mol}^{-1}$.

\begin{tabular}{|c|c|c|c|c|c|c|c|c|c|c|c|c|c|c|c|c|}
\hline \multirow{2}{*}{$\begin{array}{l}\text { Method } \\
\text { Basis Set }\end{array}$} & \multicolumn{2}{|c|}{ M06-2X } & \multicolumn{2}{|c|}{$\omega B 97 X D$} & \multicolumn{2}{|c|}{ CAM-B3LYP-D3 } & \multicolumn{2}{|c|}{ B3LYP-D3 } & \multicolumn{2}{|c|}{ M11 } & \multicolumn{2}{|c|}{ LC- $\omega$ PBE-D3 } & \multicolumn{2}{|c|}{ BLYP-D3 } & \multicolumn{2}{|c|}{ PBEPBE-D3 } \\
\hline & Uncorr. & Corr. & Uncorr. & Corr. & Uncorr. & Corr. & Uncorr. & Corr. & Uncorr. & Corr. & Uncorr. & Corr. & Uncorr & Corr. & Uncorr & Corr. \\
\hline $6-311 G(d)$ & -26.7 & -21.5 & -21.0 & -15.6 & -26.2 & -20.1 & -23.5 & -17.4 & -25.5 & -20.0 & -25.4 & -19.7 & -20.6 & -13.8 & -21.4 & -15.1 \\
\hline $6-311+G(d)$ & -25.6 & -21.0 & -20.1 & -15.4 & -24.5 & -20.1 & -21.7 & -17.4 & -24.5 & -19.2 & -24.4 & -19.4 & -18.2 & -14.0 & -19.7 & -15.1 \\
\hline $6-311 G(2 d)$ & -23.4 & -17.4 & -19.2 & -13.3 & -23.8 & -16.9 & -21.5 & -14.8 & -22.3 & -16.1 & -22.8 & -16.4 & -19.0 & -11.7 & -19.6 & -12.8 \\
\hline $6-311+G(2 d)$ & -20.5 & -17.8 & -16.6 & -13.7 & -20.2 & -17.6 & -17.9 & -15.4 & -19.7 & -16.2 & -19.7 & -16.8 & -14.8 & -12.3 & -16.0 & -13.4 \\
\hline cc-pVDZ & -25.4 & -19.3 & -20.4 & -14.3 & -25.3 & -17.8 & -22.8 & -15.5 & -24.1 & -17.4 & -24.5 & -17.7 & -20.7 & -12.2 & -20.6 & -13.3 \\
\hline jul-cc-pVDZ & -20.5 & -17.7 & -16.5 & -13.5 & -19.7 & -16.9 & -17.4 & -14.8 & -19.9 & -16.1 & -19.5 & -16.3 & -14.3 & -11.8 & -15.7 & -13.0 \\
\hline cc-pVTZ & -19.6 & -17.5 & -15.1 & -12.9 & -19.7 & -17.2 & -17.6 & -15.1 & -18.4 & -15.6 & -15.9 & -18.2 & -15.1 & -12.1 & -15.5 & -12.8 \\
\hline jul-cc-pVTZ & -18.2 & -17.2 & -13.6 & -12.7 & -17.9 & -17.1 & -15.8 & -15.0 & -16.6 & -14.5 & -16.5 & -15.6 & -13.0 & -12.1 & -13.7 & -12.8 \\
\hline Def2-SVP & -27.9 & -21.4 & -22.6 & -16.1 & -27.5 & -19.6 & -24.6 & -17.0 & -26.5 & -19.4 & -27.2 & -20.0 & -22.2 & -13.3 & -22.4 & -14.6 \\
\hline Def2-TZV & -25.8 & -20.3 & -19.5 & -14.1 & -25.1 & -19.9 & -22.4 & -17.3 & -25.7 & -19.4 & -23.6 & -17.9 & -18.6 & -13.7 & -19.2 & -14.1 \\
\hline Def2-TZVP & -18.4 & -17.3 & -14.0 & -12.8 & -17.3 & -18.5 & -16.4 & -15.2 & -17.9 & -15.9 & -17.0 & -15.8 & -13.8 & -12.3 & -14.4 & -12.9 \\
\hline Def2-QZV & -25.0 & -19.3 & -18.9 & -13.2 & -24.9 & -19.2 & -22.1 & -16.7 & -23.4 & -16.9 & -23.5 & -17.3 & -18.5 & -13.3 & -19.2 & -13.7 \\
\hline DGDZVP & -25.6 & -22.6 & -19.3 & -16.4 & -22.6 & -20.1 & -20.0 & -17.5 & -22.9 & -19.8 & -22.5 & -19.6 & -16.5 & -14.0 & -17.9 & -15.4 \\
\hline DGTZVP & -22.2 & -19.7 & -16.6 & -14.2 & -21.6 & -19.4 & -19.1 & -16.9 & -21.4 & -18.5 & -20.3 & -17.9 & -15.9 & -13.7 & -16.8 & -14.5 \\
\hline LANL2DZ & -29.2 & -22.9 & -23.0 & -16.5 & -27.1 & -20.8 & -23.8 & -17.8 & -28.9 & -21.5 & -26.9 & -19.9 & -20.0 & -13.8 & -20.8 & -14.7 \\
\hline
\end{tabular}


Table S10. CP-corrected and uncorrected interaction energy for Int.4-Cl (kJ mol$\left.{ }^{-1}\right)$ as a function of method and basis set. The benchmark $\operatorname{CCSD}(\mathrm{T}) / \mathrm{CBS}$ value for this interaction is $-8.2 \mathrm{~kJ} \mathrm{~mol}^{-1}$.

\begin{tabular}{|c|c|c|c|c|c|c|c|c|c|c|c|c|c|c|c|c|}
\hline \multirow{2}{*}{$\begin{array}{l}\text { Method } \\
\text { Basis Set }\end{array}$} & \multicolumn{2}{|c|}{ M06-2X } & \multicolumn{2}{|c|}{$\omega B 97 X D$} & \multicolumn{2}{|c|}{ CAM-B3LYP-D3 } & \multicolumn{2}{|c|}{ B3LYP-D3 } & \multicolumn{2}{|c|}{ M11 } & \multicolumn{2}{|c|}{ LC- $\omega$ PBE-D3 } & \multicolumn{2}{|c|}{ BLYP-D3 } & \multicolumn{2}{|c|}{ PBEPBE-D3 } \\
\hline & Uncorr. & Corr. & Uncorr. & Corr. & Uncorr. & Corr. & Uncorr. & Corr. & Uncorr. & Corr. & Uncorr. & Corr. & Uncorr & Corr. & Uncorr & Corr. \\
\hline $6-311 G(d)$ & -7.8 & -5.7 & -6.7 & -4.6 & -10.0 & -7.7 & -9.6 & -7.3 & -6.2 & -4.0 & -8.4 & -6.2 & -9.1 & -6.7 & -9.4 & -7.1 \\
\hline $6-311+G(d)$ & -8.5 & -5.3 & -7.2 & -4.1 & -10.1 & -7.3 & -9.7 & -6.9 & -7.6 & -3.6 & -9.0 & -5.9 & -8.9 & -6.2 & -9.5 & -6.6 \\
\hline $6-311 G(2 d)$ & -7.9 & -5.3 & -7.2 & -4.6 & -10.2 & -7.3 & -9.9 & -7.1 & -6.3 & -3.6 & -8.7 & -6.0 & -9.5 & -6.4 & -9.7 & -6.9 \\
\hline $6-311+G(2 d)$ & -7.2 & -5.1 & -6.6 & -4.4 & -9.2 & -7.2 & -8.9 & -6.9 & -6.5 & -3.5 & -8.0 & -5.9 & -8.2 & -6.3 & -8.7 & -6.7 \\
\hline $6-311 G(d f)$ & -7.4 & -5.4 & -6.4 & -4.2 & -9.7 & -7.3 & -9.3 & -7.0 & -5.9 & -3.6 & -8.1 & -5.9 & -8.9 & -6.4 & -9.2 & -6.8 \\
\hline $6-311+G(d f)$ & -8.1 & -5.0 & -6.9 & -3.8 & -9.9 & -7.0 & -9.5 & -6.6 & -7.3 & -3.3 & -8.7 & -5.5 & -8.7 & -6.0 & -9.2 & -6.4 \\
\hline $6-311 G(2 d f)$ & -7.8 & -5.1 & -7.0 & -4.4 & -10.1 & -7.1 & -9.8 & -6.9 & -6.2 & -3.4 & -8.6 & -5.7 & -9.3 & -6.3 & -9.6 & -6.7 \\
\hline $6-311+G(2 d f)$ & -7.1 & -4.9 & -6.4 & -4.2 & -9.0 & -7.0 & -8.7 & -6.8 & -6.4 & -3.3 & -7.8 & -5.6 & -8.0 & -6.2 & -8.5 & -6.5 \\
\hline $6-311 G(3 d f)$ & -7.0 & -4.5 & -6.4 & -3.8 & -9.8 & -6.9 & -9.5 & -6.7 & -5.1 & -2.4 & -8.2 & -5.4 & -9.2 & -6.2 & -9.3 & -6.5 \\
\hline $6-311+G(3 d f)$ & -6.1 & -4.4 & -5.5 & -3.8 & -8.4 & -6.9 & -8.2 & -6.7 & -4.7 & -2.5 & -7.1 & -5.3 & -7.6 & -6.1 & -8.0 & -6.4 \\
\hline cc-pVDZ & -7.9 & -5.2 & -6.9 & -4.3 & -10.4 & -6.9 & -10.1 & -6.8 & -6.5 & -3.6 & -8.8 & -5.9 & -10.3 & -6.3 & -9.6 & -6.3 \\
\hline jul-cc-pVDZ & -6.6 & -5.2 & -5.8 & -4.3 & -8.0 & -6.7 & -7.7 & -6.5 & -5.6 & -3.4 & -7.2 & -5.6 & -7.0 & -5.8 & -7.7 & -6.3 \\
\hline cc-pVTZ & -5.5 & -4.5 & -4.8 & -3.8 & -8.0 & -6.7 & -7.8 & -6.6 & -4.1 & -2.9 & -6.4 & -5.3 & -6.1 & -6.1 & -7.5 & -6.2 \\
\hline jul-cc-pVTZ & -4.9 & -4.4 & -4.0 & -3.6 & -7.1 & -6.7 & -6.9 & -6.6 & -3.3 & -2.2 & -5.6 & -5.2 & -6.4 & -6.1 & -6.7 & -6.3 \\
\hline cc-pVQZ & -4.7 & -4.3 & -4.0 & -3.5 & -7.2 & -6.7 & -7.1 & -6.6 & -2.8 & -2.1 & -5.6 & -5.1 & -6.7 & -6.1 & -6.9 & -6.3 \\
\hline jul-cc-pVQZ & -4.5 & -4.4 & -3.7 & -3.6 & -6.9 & -6.8 & -6.8 & -6.7 & -2.3 & -1.8 & -5.4 & -5.2 & -6.3 & -6.2 & -6.5 & -6.4 \\
\hline Def2-SVP & -7.7 & -4.6 & -6.4 & -3.4 & -10.3 & -6.4 & -10.0 & -6.2 & -6.3 & -2.9 & -8.7 & -5.5 & -10.2 & -5.7 & -9.2 & -5.5 \\
\hline Def2-TZV & -5.6 & -3.8 & -4.4 & -2.6 & -8.1 & -6.2 & -7.9 & -6.1 & -4.5 & -2.2 & -6.4 & -4.4 & -7.5 & -5.6 & -7.4 & -5.6 \\
\hline Def2-TZVP & -5.1 & -4.5 & -4.4 & -3.7 & -7.7 & -6.9 & -7.5 & -6.7 & -3.9 & -2.9 & -6.0 & -5.3 & -7.2 & -6.2 & -7.2 & -6.3 \\
\hline Def2-QZV & -5.2 & -3.6 & -4.1 & -2.4 & -7.7 & -6.1 & -7.5 & -6.0 & -3.3 & -1.2 & -6.2 & -4.4 & -7.1 & -5.5 & -7.2 & -5.6 \\
\hline Def2-QZVP & -4.5 & -4.4 & -3.8 & -3.6 & -7.0 & -6.8 & -6.9 & -6.7 & -2.3 & -1.9 & -5.4 & -5.3 & -6.4 & -6.2 & -6.7 & -6.4 \\
\hline DGDZVP & -6.9 & -5.6 & -5.4 & -4.3 & -8.2 & -7.2 & -7.9 & -6.9 & -4.8 & -3.6 & -7.0 & -5.9 & -7.4 & -6.3 & -7.6 & -6.6 \\
\hline DGDZVP2 & -6.8 & -5.3 & -5.3 & -4.0 & -8.3 & -7.1 & -8.0 & -6.8 & -4.8 & -3.3 & -6.9 & -5.6 & -7.7 & -6.2 & -7.7 & -6.4 \\
\hline DGTZVP & -5.6 & -4.7 & -4.5 & -3.6 & -7.9 & -7.0 & -7.7 & -6.8 & -4.2 & -3.1 & -6.3 & -5.4 & -7.2 & -6.3 & -7.4 & -6.4 \\
\hline LANL2DZ & -5.9 & -3.9 & -4.7 & -2.8 & -7.9 & -5.7 & -7.7 & -5.5 & -4.9 & -2.8 & -6.4 & -4.4 & -7.7 & -5.1 & -7.2 & -5.1 \\
\hline
\end{tabular}


Table S11. CP-corrected and uncorrected interaction energy for Int.4-Br $\left(\mathrm{kJ} \mathrm{mol}^{-1}\right)$ as a function of method and basis set. The benchmark $\operatorname{CCSD}(\mathrm{T}) / \mathrm{CBS}$ value for this interaction is $-10.8 \mathrm{~kJ} \mathrm{~mol}^{-1}$.

\begin{tabular}{|c|c|c|c|c|c|c|c|c|c|c|c|c|c|c|c|c|}
\hline \multirow{2}{*}{$\begin{array}{l}\text { Method } \\
\text { Basis Set }\end{array}$} & \multicolumn{2}{|c|}{ M06-2X } & \multicolumn{2}{|c|}{$\omega B 97 X D$} & \multicolumn{2}{|c|}{ CAM-B3LYP-D3 } & \multicolumn{2}{|c|}{ B3LYP-D3 } & \multicolumn{2}{|c|}{ M11 } & \multicolumn{2}{|c|}{ LC- $\omega$ PBE-D3 } & \multicolumn{2}{|c|}{ BLYP-D3 } & \multicolumn{2}{|c|}{ PBEPBE-D3 } \\
\hline & Uncorr. & Corr. & Uncorr. & Corr. & Uncorr. & Corr. & Uncorr. & Corr. & Uncorr. & Corr. & Uncorr. & Corr. & Uncorr & Corr. & Uncorr & Corr. \\
\hline $6-311 G(d)$ & -10.4 & -8.4 & -10.0 & -8.1 & -13.3 & -10.9 & -13.1 & -10.8 & -8.1 & -6.1 & -11.9 & -9.7 & -12.7 & -10.1 & -12.4 & -10.0 \\
\hline $6-311+G(d)$ & -10.3 & -8.1 & -9.9 & -7.7 & -12.6 & -10.5 & -12.4 & -10.3 & -8.4 & -5.6 & -11.7 & -9.2 & -11.5 & -9.5 & -11.8 & -9.5 \\
\hline $6-311 G(2 d)$ & -9.4 & -6.5 & -9.6 & -6.7 & -12.7 & -9.3 & -12.6 & -9.4 & -7.1 & -4.2 & -11.2 & -8.1 & -12.4 & -8.8 & -12.0 & -8.7 \\
\hline $6-311+G(2 d)$ & -8.2 & -6.6 & -8.5 & -6.7 & -11.0 & -9.3 & -10.9 & -9.3 & -6.3 & -4.1 & -9.9 & -8.0 & -10.2 & -8.7 & -10.3 & -8.6 \\
\hline $6-311 G(d f)$ & -9.8 & -7.8 & -9.5 & -7.4 & -12.7 & -10.3 & -12.6 & -10.2 & -7.5 & -5.4 & -11.3 & -9.1 & -12.2 & -9.6 & -11.9 & -9.5 \\
\hline $6-311 G(2 d f)$ & -9.1 & -6.3 & -9.3 & -6.4 & -12.5 & -9.1 & -12.4 & -9.2 & -6.9 & -4.0 & -10.9 & -7.8 & -12.1 & -8.6 & -11.7 & -8.5 \\
\hline $6-311+G(2 d f)$ & -7.8 & -6.4 & -8.1 & -6.5 & -10.6 & -9.1 & -10.6 & -9.2 & -6.2 & -4.0 & -9.5 & -7.8 & -9.9 & -8.5 & -11.4 & -8.4 \\
\hline $6-311 G(3 d f)$ & -8.9 & -6.2 & -8.9 & -6.3 & -12.1 & -9.1 & -12.0 & -9.2 & -6.4 & -3.6 & -10.6 & -7.7 & -11.8 & -8.6 & -11.4 & -8.4 \\
\hline $6-311+G(3 d f)$ & -7.3 & -6.2 & -7.4 & -6.3 & -10.0 & -9.0 & -10.0 & -9.0 & -5.2 & -3.6 & -8.8 & -7.6 & -9.4 & -8.5 & -9.4 & -8.4 \\
\hline cc-pVDZ & -11.3 & -8.2 & -10.9 & -7.9 & -14.1 & -10.1 & -14.1 & -10.2 & -9.2 & -5.9 & -12.7 & -9.4 & -14.2 & -9.6 & -13.1 & -9.3 \\
\hline jul-cc-pVDZ & -9.7 & -7.5 & -9.3 & -7.2 & -10.9 & -9.2 & -10.9 & -9.1 & -7.8 & -5.0 & -10.5 & -8.4 & -10.1 & -8.4 & -10.4 & -8.6 \\
\hline jul-cc-pVTZ & -7.1 & -6.4 & -6.9 & -6.3 & -9.6 & -9.1 & -9.7 & -9.2 & -5.2 & -3.6 & -8.2 & -7.7 & -9.1 & -8.6 & -9.0 & -8.5 \\
\hline cc-pVQZ & -6.8 & -6.3 & -6.7 & -6.2 & -9.6 & -9.0 & -9.7 & -9.1 & -4.3 & -3.3 & -8.1 & -7.6 & -9.3 & -8.6 & -9.0 & -8.4 \\
\hline jul-cc-pVQZ & -6.7 & -6.5 & -6.5 & -6.3 & -9.3 & -9.1 & -9.4 & -9.2 & -3.8 & -3.2 & -7.9 & -7.7 & -8.9 & -8.7 & -8.7 & -8.5 \\
\hline Def2-SVP & -11.9 & -8.5 & -11.3 & -8.0 & -14.5 & -10.3 & -14.4 & -10.3 & -9.8 & -6.2 & -13.3 & -9.8 & -14.5 & -9.5 & -13.4 & -9.3 \\
\hline Def2-TZV & -10.7 & -8.7 & -9.7 & -7.8 & -13.2 & -11.3 & -12.8 & -11.1 & -9.2 & -6.6 & -11.8 & -9.7 & -12.1 & -10.3 & -11.8 & -10.0 \\
\hline Def2-TZVP & -7.1 & -6.3 & -6.9 & -6.2 & -9.9 & -9.0 & -9.9 & -9.0 & -5.4 & -4.1 & -8.5 & -7.6 & -9.5 & -8.5 & -9.2 & -8.2 \\
\hline Def2-QZV & -11.3 & -9.5 & -10.8 & -8.9 & -14.1 & -12.3 & -13.9 & -12.1 & -8.7 & -6.2 & -12.8 & -10.8 & -13.1 & -11.3 & -12.9 & -11.1 \\
\hline Def2-QZVP & -6.5 & -6.3 & -6.4 & -6.2 & -9.2 & -9.0 & -9.3 & -9.1 & -3.6 & -3.1 & -7.8 & -7.6 & -8.9 & -8.6 & -8.7 & -8.4 \\
\hline DGDZVP & -10.2 & -8.6 & -9.7 & -8.3 & -12.0 & -10.5 & -11.8 & -10.5 & -6.9 & -5.3 & -11.1 & -9.6 & -11.2 & -9.7 & -11.3 & -9.8 \\
\hline LANL2DZ & -11.9 & -9.4 & -10.1 & -7.7 & -13.2 & -10.5 & -12.9 & -10.3 & -10.3 & -7.7 & -11.9 & -9.3 & -12.5 & -9.5 & -11.8 & -9.2 \\
\hline
\end{tabular}


Table S12. CP-corrected and uncorrected interaction energy for Int.4-F ( $\left.\mathrm{kJ} \mathrm{mol}^{-1}\right)$ as a function of method and basis set. The benchmark $\mathrm{CCSD}(\mathrm{T}) / \mathrm{CBS}$ value for this interaction is $-2.4 \mathrm{~kJ} \mathrm{~mol}^{-1}$.

\begin{tabular}{|c|c|c|c|c|c|c|c|c|c|c|c|c|c|c|c|c|}
\hline \multirow{2}{*}{$\begin{array}{l}\text { Method } \\
\text { Basis Set }\end{array}$} & \multicolumn{2}{|c|}{ M06-2X } & \multicolumn{2}{|c|}{$\omega B 97 X D$} & \multicolumn{2}{|c|}{ CAM-B3LYP-D3 } & \multicolumn{2}{|c|}{ B3LYP-D3 } & \multicolumn{2}{|c|}{ M11 } & \multicolumn{2}{|c|}{ LC- $\omega$ PBE-D3 } & \multicolumn{2}{|c|}{ BLYP-D3 } & \multicolumn{2}{|c|}{ PBEPBE-D3 } \\
\hline & Uncorr. & Corr. & Uncorr. & Corr. & Uncorr. & Corr. & Uncorr. & Corr. & Uncorr. & Corr. & Uncorr. & Corr. & Uncorr & Corr. & Uncorr & Corr. \\
\hline $6-311 G(d)$ & -2.1 & 0.3 & -2.3 & 0.0 & -4.3 & -1.4 & -4.1 & -1.3 & -1.3 & 1.3 & -4.3 & -1.4 & -4.7 & -1.3 & -5.1 & -2.1 \\
\hline $6-311 G(2 d)$ & -2.8 & -0.3 & -3.0 & -0.5 & -4.9 & -1.8 & -4.6 & -1.7 & -2.1 & 0.7 & -4.1 & -1.1 & -5.1 & -1.7 & -5.6 & -2.5 \\
\hline $6-311+G(2 d)$ & -1.6 & -0.3 & -1.8 & -0.5 & -2.8 & -1.7 & -2.6 & -1.5 & -0.9 & 0.8 & -2.4 & -1.0 & -2.3 & -1.3 & -3.6 & -2.5 \\
\hline cc-pVDZ & -2.0 & 0.2 & -2.3 & -0.3 & -4.1 & -1.6 & -4.1 & -1.7 & -1.4 & 1.0 & -3.3 & -1.0 & -4.7 & -1.9 & -4.7 & -2.2 \\
\hline jul-cc-pVDZ & -1.3 & -0.4 & -1.5 & -0.5 & -2.5 & -1.5 & -2.5 & -1.5 & -0.5 & 0.8 & -2.2 & -1.1 & -2.2 & -1.3 & -3.5 & -2.6 \\
\hline cc-pVTZ & -1.3 & 0.0 & -1.7 & -0.5 & -3.1 & -1.6 & -2.9 & -1.4 & -0.8 & 0.7 & -2.5 & -1.0 & -3.1 & -1.3 & -3.9 & -2.3 \\
\hline jul-cc-pVTZ & -0.6 & -0.2 & -0.9 & -0.5 & -2.0 & -1.6 & -1.8 & -1.4 & 0.0 & 0.9 & -1.4 & -1.0 & -1.5 & -1.2 & -2.8 & -2.4 \\
\hline Def2-SVP & -1.3 & 0.5 & -1.7 & 0.1 & -3.4 & -1.3 & -3.4 & -1.3 & -0.9 & 1.2 & -2.7 & -0.7 & -4.0 & -1.5 & -4.0 & -1.8 \\
\hline Def2-TZV & 0.2 & 1.5 & -0.2 & 1.1 & -1.6 & -0.2 & -1.5 & -0.1 & 1.0 & 2.5 & -1.0 & 0.5 & -1.5 & -0.1 & -2.7 & -1.3 \\
\hline Def2-QZV & -0.3 & 1.6 & -0.9 & 1.1 & -2.2 & -0.3 & -2.1 & -0.3 & 0.6 & 3.0 & -1.6 & 0.5 & -2.1 & -0.3 & -3.3 & -1.4 \\
\hline DGDZVP & -1.8 & -1.1 & -1.2 & -0.4 & -2.5 & -1.5 & -2.1 & -1.3 & -0.3 & 0.5 & -1.9 & -1.0 & -2.3 & -1.1 & -3.2 & -2.2 \\
\hline LANL2DZ & 0.3 & 1.5 & -0.1 & 1.2 & -1.7 & 0.0 & -1.6 & 0.0 & 0.9 & 2.4 & -1.0 & 0.7 & -2.2 & -0.1 & -2.8 & -1.0 \\
\hline
\end{tabular}


Table S13. CP-corrected and uncorrected interaction energy for Int.5-Cl $\left(\mathrm{kJ} \mathrm{mol}^{-1}\right)$ as a function of method and basis set. The benchmark $\mathrm{CCSD}(\mathrm{T}) / \mathrm{CBS}$ value for this interaction is $-4.1 \mathrm{~kJ} \mathrm{~mol}^{-1}$.

\begin{tabular}{|c|c|c|c|c|c|c|c|c|c|c|c|c|c|c|c|c|}
\hline \multirow{2}{*}{$\begin{array}{l}\text { Method } \\
\text { Basis Set }\end{array}$} & \multicolumn{2}{|c|}{ M06-2X } & \multicolumn{2}{|c|}{$\omega B 97 X D$} & \multicolumn{2}{|c|}{ CAM-B3LYP-D3 } & \multicolumn{2}{|c|}{ B3LYP-D3 } & \multicolumn{2}{|c|}{ M11 } & \multicolumn{2}{|c|}{ LC- $\omega$ PBE-D3 } & \multicolumn{2}{|c|}{ BLYP-D3 } & \multicolumn{2}{|c|}{ PBEPBE-D3 } \\
\hline & Uncorr. & Corr. & Uncorr. & Corr. & Uncorr. & Corr. & Uncorr. & Corr. & Uncorr. & Corr. & Uncorr. & Corr. & Uncorr & Corr. & Uncorr & Corr. \\
\hline $6-311 G(d)$ & -3.9 & -1.3 & -4.0 & -1.5 & -5.4 & -2.8 & -5.6 & -3.1 & -3.0 & 0.0 & -4.9 & -2.3 & -5.2 & -2.6 & -5.9 & -3.5 \\
\hline $6-311+G(d)$ & -4.1 & -1.2 & -4.2 & -1.3 & -5.4 & -2.8 & -5.5 & -2.9 & -4.0 & 0.0 & -5.1 & -2.3 & -4.9 & -2.4 & -5.9 & -3.4 \\
\hline $6-311 G(2 d)$ & -3.8 & -1.0 & -4.2 & -1.5 & -5.6 & -2.8 & -5.8 & -3.1 & -2.8 & 0.3 & -5.0 & -2.3 & -5.4 & -2.7 & -6.2 & -3.6 \\
\hline $6-311+G(2 d)$ & -3.4 & -1.2 & -3.8 & -1.5 & -5.0 & -2.9 & -5.1 & -3.1 & -3.3 & 0.1 & -4.6 & -2.4 & -4.6 & -2.6 & -5.5 & -3.6 \\
\hline 6-311G(df) & -4.1 & -1.4 & -3.8 & -1.5 & -5.6 & -2.9 & -5.8 & -3.1 & -3.2 & -0.1 & -5.1 & -2.4 & -5.3 & -2.7 & -6.1 & -3.6 \\
\hline $6-311+G(d f)$ & -4.2 & -1.3 & -4.3 & -1.5 & -5.4 & -2.8 & -5.5 & -3.0 & -4.1 & -0.2 & -5.1 & -2.4 & -4.9 & -2.5 & -5.9 & -3.4 \\
\hline $6-311 G(2 d f)$ & -4.1 & -1.2 & -4.4 & -1.5 & -5.7 & -2.9 & -5.9 & -3.1 & -3.1 & 0.2 & -5.2 & -2.4 & -5.5 & -2.7 & -6.3 & -3.6 \\
\hline $6-311+G(2 d f)$ & -3.6 & -1.3 & -3.8 & -1.6 & -5.0 & -2.9 & -5.1 & -3.1 & -3.5 & 0.0 & -4.7 & -2.5 & -4.6 & -2.6 & -5.5 & -3.6 \\
\hline $6-311 G(3 d f)$ & -3.3 & -0.9 & -3.8 & -1.3 & -5.3 & -2.8 & -5.5 & -3.0 & -2.4 & 0.6 & -4.8 & -2.3 & -5.2 & -2.6 & -6.0 & -3.5 \\
\hline $6-311+G(3 d f)$ & -3.1 & -1.0 & -3.4 & -1.3 & -4.6 & -2.8 & -4.8 & -3.0 & -2.7 & 0.4 & -4.4 & -2.3 & -4.3 & -2.6 & -5.2 & -3.5 \\
\hline cc-pVDZ & -2.3 & -0.4 & -2.6 & -0.9 & -4.4 & -2.2 & -4.5 & -2.4 & -1.4 & 0.5 & -3.9 & -2.1 & -4.6 & -2.1 & -4.6 & -2.5 \\
\hline jul-cc-pVDZ & -2.3 & -1.4 & -2.6 & -1.6 & -3.6 & -2.7 & -3.6 & -2.8 & -1.5 & 0.0 & -3.6 & -2.6 & -3.1 & -2.3 & -4.2 & -3.3 \\
\hline cc-pVTZ & -1.6 & -0.6 & -2.1 & -1.0 & -3.8 & -2.6 & -4.0 & -2.9 & -0.6 & 0.6 & -3.2 & -2.2 & -3.9 & -2.6 & -4.4 & -3.2 \\
\hline jul-cc-pVTZ & -1.2 & -0.7 & -1.5 & -1.1 & -3.1 & -2.7 & -3.3 & -2.9 & -0.3 & 1.1 & -2.6 & -2.2 & -2.9 & -2.6 & -3.7 & -3.4 \\
\hline cc-pVQZ & -0.8 & -0.4 & -1.3 & -0.9 & -3.2 & -2.7 & -3.5 & -3.0 & 0.8 & 1.5 & -2.6 & -2.1 & -3.3 & -2.6 & -4.0 & -3.4 \\
\hline jul-cc-pVQZ & -0.6 & -0.5 & -1.1 & -1.0 & -2.8 & -2.7 & -3.1 & -3.0 & 1.1 & 1.6 & -2.3 & -2.2 & -2.8 & -2.7 & -3.6 & -3.5 \\
\hline Def2-SVP & -3.3 & 0.5 & -3.2 & 0.5 & -6.4 & -1.9 & -6.5 & -2.1 & -2.3 & 1.8 & -5.3 & -1.5 & -6.9 & -2.0 & -6.1 & -1.8 \\
\hline Def2-TZV & -1.2 & 0.0 & -1.6 & -0.4 & -3.5 & -2.2 & -3.7 & -2.4 & -0.1 & 1.2 & -3.0 & -1.7 & -3.4 & -1.9 & -4.0 & -2.6 \\
\hline Def2-TZVP & -1.3 & -0.6 & -1.8 & -1.0 & -3.6 & -2.7 & -3.8 & -2.9 & -0.3 & 0.7 & -2.9 & -2.2 & -3.7 & -2.6 & -4.4 & -3.3 \\
\hline Def2-QZV & -0.5 & 0.5 & -0.9 & 0.1 & -3.1 & -2.1 & -3.2 & -2.2 & 1.0 & 2.5 & -2.5 & -1.4 & -2.8 & -1.8 & -3.5 & -2.4 \\
\hline Def2-QZVP & -0.6 & -0.5 & -1.1 & -1.0 & -2.9 & -2.7 & -3.2 & -3.0 & 1.3 & 1.6 & -2.3 & -2.2 & -2.9 & -2.6 & -3.7 & -3.4 \\
\hline DGDZVP & -2.0 & -1.0 & -2.1 & -1.2 & -3.4 & -2.5 & -3.5 & -2.6 & -0.7 & 0.3 & -3.2 & -2.3 & -3.1 & -2.2 & -3.9 & -3.0 \\
\hline DGDZVP2 & -1.8 & -0.9 & -2.0 & -1.1 & -3.3 & -2.5 & -3.4 & -2.7 & -0.3 & 0.5 & -3.0 & -2.2 & -3.1 & -2.2 & -3.8 & -3.1 \\
\hline DGTZVP & -1.0 & -0.2 & -1.5 & -0.7 & -3.3 & -2.4 & -3.5 & -2.6 & 0.0 & 0.9 & -2.7 & -1.9 & -3.1 & -2.2 & -3.8 & -3.0 \\
\hline LANL2DZ & -1.3 & 0.2 & -2.1 & -0.3 & -4.1 & -1.8 & -3.9 & -1.7 & -1.6 & 0.4 & -3.8 & -1.9 & -3.8 & -1.1 & -3.9 & -1.7 \\
\hline
\end{tabular}


Table S14. CP-corrected and uncorrected interaction energy for Int.5-Br $\left(\mathrm{kJ} \mathrm{mol}^{-1}\right)$ as a function of method and basis set. The benchmark $\mathrm{CCSD}(\mathrm{T}) / \mathrm{CBS}$ value for this interaction is $-4.4 \mathrm{~kJ} \mathrm{~mol}^{-1}$.

\begin{tabular}{|c|c|c|c|c|c|c|c|c|c|c|c|c|c|c|c|c|}
\hline \multirow{2}{*}{$\begin{array}{l}\text { Method } \\
\text { Basis Set }\end{array}$} & \multicolumn{2}{|c|}{ M06-2X } & \multicolumn{2}{|c|}{$\omega B 97 X D$} & \multicolumn{2}{|c|}{ CAM-B3LYP-D3 } & \multicolumn{2}{|c|}{ B3LYP-D3 } & \multicolumn{2}{|c|}{ M11 } & \multicolumn{2}{|c|}{ LC- $\omega$ PBE-D3 } & \multicolumn{2}{|c|}{ BLYP-D3 } & \multicolumn{2}{|c|}{ PBEPBE-D3 } \\
\hline & Uncorr. & Corr. & Uncorr. & Corr. & Uncorr. & Corr. & Uncorr. & Corr. & Uncorr. & Corr. & Uncorr. & Corr. & Uncorr & Corr. & Uncorr & Corr. \\
\hline $6-311 G(d)$ & -1.5 & 0.0 & -2.6 & -1.2 & -3.7 & -2.1 & -4.4 & -2.8 & -0.3 & 1.3 & -3.6 & -2.2 & -4.1 & -2.3 & -4.3 & -2.6 \\
\hline $6-311+G(d)$ & -1.8 & -0.2 & -2.8 & -1.3 & -3.6 & -2.2 & -4.2 & -2.8 & -1.2 & 1.2 & -3.8 & -2.2 & -3.6 & -2.2 & -4.3 & -2.8 \\
\hline $6-311 G(2 d)$ & -1.9 & -0.1 & -3.3 & -1.5 & -4.4 & -2.4 & -5.1 & -3.1 & -0.5 & 1.5 & -4.2 & -2.4 & -4.9 & -2.7 & -5.2 & -3.1 \\
\hline $6-311+G(2 d)$ & -1.8 & -0.4 & -3.2 & -1.7 & -4.0 & -2.5 & -4.6 & -3.2 & -0.8 & 1.4 & -4.1 & -2.5 & -4.0 & -2.7 & -4.7 & -3.3 \\
\hline $6-311 G(d f)$ & -1.8 & -0.3 & -2.8 & -1.4 & -3.9 & -2.3 & -4.5 & -2.9 & -0.6 & 1.0 & -3.8 & -2.3 & -4.2 & -2.3 & -4.5 & -2.8 \\
\hline $6-311+G(d f)$ & -2.0 & -0.4 & -3.0 & -1.5 & -3.7 & -2.3 & -4.3 & -2.9 & -1.3 & 1.0 & -3.9 & -2.4 & -3.6 & -2.3 & -4.3 & -2.9 \\
\hline $6-311 G(2 d f)$ & -2.2 & -0.3 & -3.5 & -1.6 & -4.5 & -2.5 & -5.2 & -3.2 & -0.7 & 1.3 & -4.4 & -2.6 & -4.9 & -2.7 & -5.3 & -3.1 \\
\hline $6-311+G(2 d f)$ & -2.0 & -0.5 & -3.3 & -1.8 & -3.9 & -2.6 & -4.6 & -3.2 & -1.0 & 1.2 & -4.1 & -2.6 & -3.9 & -2.7 & -4.6 & -3.3 \\
\hline $6-311 G(3 d f)$ & -2.2 & -0.5 & -3.5 & -1.7 & -4.6 & -2.6 & -5.3 & -3.3 & -0.9 & 1.2 & -4.4 & -2.6 & -5.0 & -2.8 & -5.4 & -3.3 \\
\hline $6-311+G(3 d f)$ & -1.5 & -0.6 & -2.7 & -1.8 & -2.6 & -3.4 & -4.1 & -3.3 & -0.6 & 1.1 & -3.6 & -2.7 & -3.5 & -2.8 & -4.1 & -3.4 \\
\hline cc-pVDZ & -2.4 & -0.5 & -3.4 & -1.6 & -4.4 & -2.0 & -4.9 & -2.6 & -1.3 & 0.8 & -4.7 & -2.8 & -4.8 & -2.1 & -4.7 & -2.4 \\
\hline jul-cc-pVDZ & -2.8 & -1.5 & -3.6 & -2.4 & -3.6 & -2.6 & -4.1 & -3.1 & -1.6 & 0.1 & -4.6 & -3.3 & -3.4 & -2.4 & -4.5 & -3.4 \\
\hline cc-pVTZ & -1.0 & -0.1 & -2.3 & -1.5 & -3.4 & -2.5 & -3.1 & -4.1 & 0.0 & 1.3 & -3.4 & -2.6 & -3.7 & -2.6 & -4.1 & -3.1 \\
\hline jul-cc-pVTZ & -0.9 & -0.4 & -2.1 & -1.7 & -3.0 & -2.6 & -4.1 & -3.1 & -0.7 & 1.4 & -3.1 & -2.7 & -3.1 & -2.6 & -3.7 & -3.3 \\
\hline cc-pVQZ & -0.7 & -0.3 & -2.1 & -1.6 & -3.1 & -2.6 & -3.7 & -3.2 & 1.1 & 1.9 & -3.1 & -2.7 & -3.3 & -2.7 & -3.9 & -3.3 \\
\hline jul-cc-pVQZ & -0.6 & -0.5 & -1.9 & -1.7 & -2.8 & -2.6 & -3.4 & -3.3 & 1.5 & 2.1 & -2.9 & -2.7 & -2.9 & -2.8 & -3.6 & -3.4 \\
\hline Def2-SVP & -3.3 & -0.8 & -3.9 & -1.3 & -5.2 & -1.9 & -5.8 & -2.5 & -1.9 & 1.0 & -5.4 & -2.8 & -5.8 & -2.0 & -5.6 & -2.3 \\
\hline Def2-TZV & -0.5 & -0.1 & -1.9 & -0.7 & -3.1 & -1.9 & -3.6 & -2.4 & 0.6 & 1.9 & -3.3 & -1.9 & -2.9 & -1.7 & -3.4 & -2.1 \\
\hline Def2-TZVP & -0.8 & -0.2 & -2.3 & -1.5 & -3.3 & -2.5 & -3.9 & -3.2 & 0.2 & 1.3 & -3.3 & -2.6 & -3.5 & -2.6 & -4.1 & -3.2 \\
\hline Def2-QZV & -0.7 & -0.2 & -2.1 & -1.1 & -3.3 & -2.3 & -3.9 & -3.0 & 1.3 & 2.5 & -3.3 & -2.3 & -3.5 & -2.5 & -4.0 & -3.0 \\
\hline Def2-QZVP & -0.4 & -0.1 & -1.8 & -1.6 & -2.8 & -2.6 & -3.5 & -3.2 & 1.7 & 2.1 & -2.9 & -2.6 & -3.0 & -2.7 & -3.6 & -3.3 \\
\hline DGDZVP & -1.5 & -0.3 & -2.4 & -1.3 & -3.3 & -2.2 & -3.9 & -2.8 & 0.5 & 1.7 & -3.5 & -2.3 & -3.4 & -2.3 & -3.8 & -2.8 \\
\hline LANL2DZ & -1.9 & -0.2 & -2.4 & -0.9 & -3.1 & -1.2 & -3.3 & -1.5 & -0.9 & 0.8 & -3.8 & -2.2 & -2.7 & -0.6 & -3.0 & -1.3 \\
\hline
\end{tabular}


Table S15. CP-corrected and uncorrected interaction energy for Int.6-CN $\left(\mathrm{kJ} \mathrm{mol}^{-1}\right)$ as a function of method and basis set. The benchmark $\operatorname{CCSD}(\mathrm{T}) / \mathrm{CBS}$ value for this interaction is $-25.9 \mathrm{~kJ} \mathrm{~mol}^{-1}$.

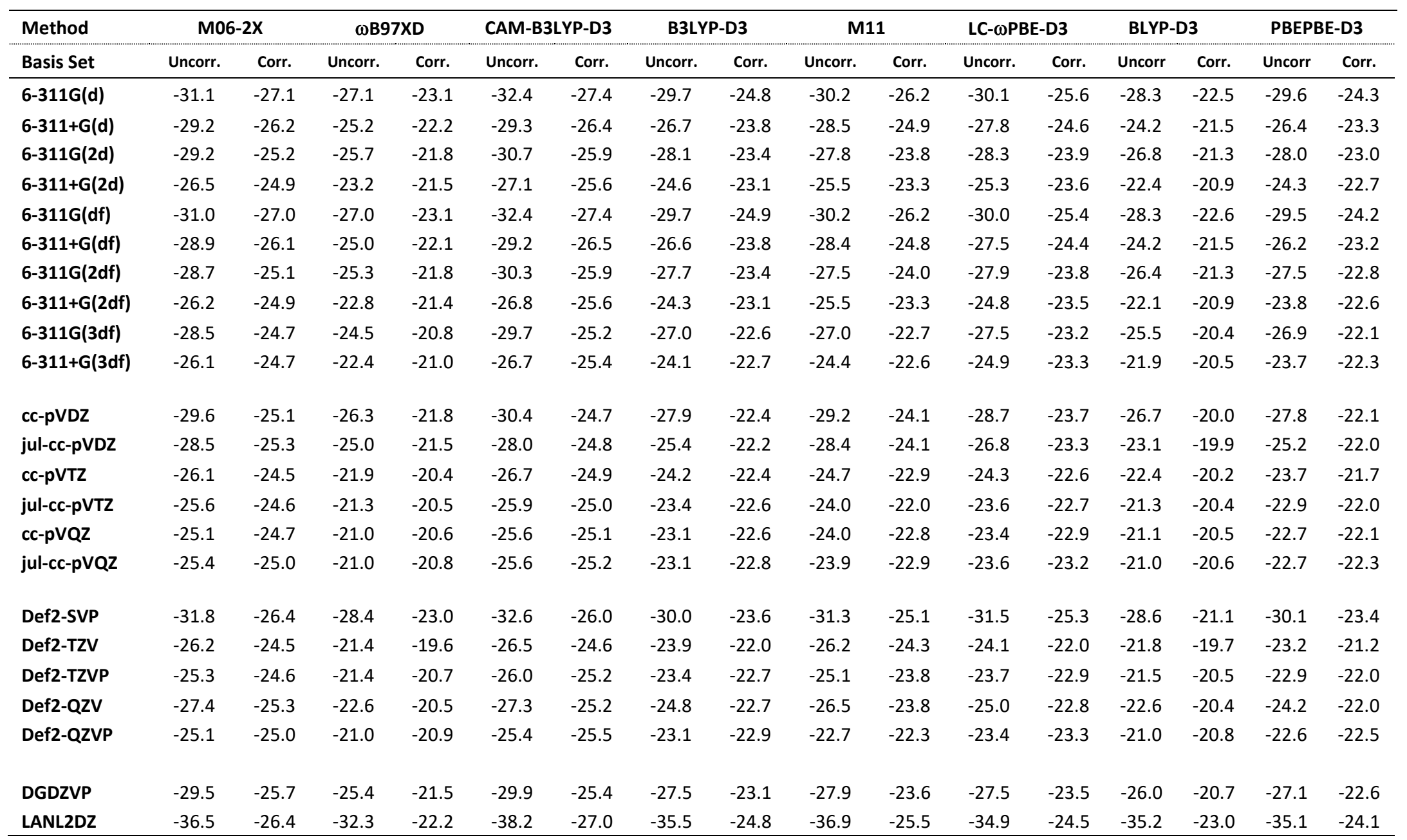


SUP-2 QTAIM Parameters at BCPs and RCPs of Int.1-X (X = F, Cl, Br) - Int.6-CN (Table S16 - S30).

Table S16. Molecular graphs and topological parameters of electron density at the BCP and RCP for Int.1-Cl.

\begin{tabular}{|c|c|c|c|c|c|c|}
\hline Name & Atoms & Rho & DelSqRho & $V(r)$ & $G(r)$ & $H(r)$ \\
\hline BCP1 & N6 - S11 & 0.013 & 0.044 & -0.008 & 0.010 & 0.001 \\
\hline BCP2 & $\mathrm{Cl} 1$ - C2 & 0.210 & -0.266 & -0.208 & 0.071 & -0.137 \\
\hline BCP3 & C2 - N6 & 0.343 & -1.060 & -0.632 & 0.184 & -0.449 \\
\hline BCP4 & C2 - N3 & 0.358 & -1.145 & -0.706 & 0.210 & -0.496 \\
\hline BCP5 & N3 - S4 & 0.224 & -0.416 & -0.347 & 0.122 & -0.225 \\
\hline BCP6 & N6 - S10 & 0.013 & 0.045 & -0.008 & 0.010 & 0.002 \\
\hline BCP7 & S5 - N6 & 0.232 & -0.447 & -0.403 & 0.146 & -0.258 \\
\hline BCP8 & S4 - S5 & 0.138 & -0.057 & -0.112 & 0.049 & -0.063 \\
\hline BCP9 & C8 - N12 & 0.344 & -1.053 & -0.629 & 0.183 & -0.446 \\
\hline ВCP10 & $\mathrm{Cl} 7$ - C8 & 0.210 & -0.269 & -0.208 & 0.071 & -0.138 \\
\hline BCP11 & S10 - S11 & 0.139 & -0.058 & -0.112 & 0.049 & -0.063 \\
\hline BCP12 & C8 - N9 & 0.358 & -1.141 & -0.707 & 0.211 & -0.496 \\
\hline BCP13 & N9 - S10 & 0.224 & -0.421 & -0.352 & 0.123 & -0.228 \\
\hline BCP14 & S11 - N12 & 0.232 & -0.449 & -0.408 & 0.148 & -0.260 \\
\hline Name & Atom Count & Rho & DelSqRho & $V(r)$ & $G(r)$ & $H(r)$ \\
\hline RCP1 & 5 & 0.039 & 0.202 & -0.049 & 0.050 & 0.001 \\
\hline $\mathrm{RCP2}$ & 5 & 0.039 & 0.202 & -0.049 & 0.050 & 0.001 \\
\hline RCP3 & 3 & 0.013 & 0.045 & -0.008 & 0.010 & 0.002 \\
\hline
\end{tabular}


Table S17. Molecular graphs and topological parameters of electron density at the $\mathrm{BCP}$ and $\mathrm{RCP}$ for Int.1-Br.

\begin{tabular}{|c|c|c|c|c|c|c|}
\hline Name & Atoms & Rho & DelSqRho & $V(r)$ & $G(r)$ & $H(r)$ \\
\hline BCP1 & S2 - S3 & 0.138 & -0.057 & -0.112 & 0.049 & -0.063 \\
\hline BCP2 & N4 - S8 & 0.013 & 0.044 & -0.008 & 0.009 & 0.001 \\
\hline BCP3 & $\mathrm{Br} 1$ - C6 & 0.168 & -0.131 & -0.156 & 0.062 & -0.094 \\
\hline BCP4 & N4 - S9 & 0.013 & 0.046 & -0.008 & 0.010 & 0.002 \\
\hline BCP5 & S2 - N4 & 0.232 & -0.447 & -0.399 & 0.144 & -0.255 \\
\hline BCP6 & S3 - N5 & 0.225 & -0.421 & -0.357 & 0.126 & -0.231 \\
\hline BCP7 & N4 - C6 & 0.338 & -1.024 & -0.613 & 0.179 & -0.435 \\
\hline BCP8 & N5 - C6 & 0.366 & -1.183 & -0.774 & 0.239 & -0.535 \\
\hline BCP9 & Br1 - S8 & 0.005 & 0.016 & -0.002 & 0.003 & 0.001 \\
\hline ВCP10 & S8 - S9 & 0.139 & -0.058 & -0.112 & 0.049 & -0.063 \\
\hline BCP11 & N10 - C12 & 0.338 & -1.017 & -0.610 & 0.178 & -0.432 \\
\hline BCP12 & S8 - N10 & 0.232 & -0.449 & -0.405 & 0.146 & -0.258 \\
\hline ВCP13 & N11 - C12 & 0.366 & -1.179 & -0.775 & 0.240 & -0.535 \\
\hline BCP14 & S9 - N11 & 0.225 & -0.425 & -0.362 & 0.128 & -0.234 \\
\hline BCP15 & Br7 - C12 & 0.168 & -0.133 & -0.154 & 0.061 & -0.094 \\
\hline Name & Atom Count & Rho & DelSqRho & $V(r)$ & $G(r)$ & $H(r)$ \\
\hline $\mathrm{RCP1}$ & 4 & 0.005 & 0.017 & -0.002 & 0.003 & 0.001 \\
\hline $\mathrm{RCP2}$ & 5 & 0.039 & 0.204 & -0.050 & 0.050 & 0.001 \\
\hline RCP3 & 3 & 0.013 & 0.044 & -0.008 & 0.010 & 0.002 \\
\hline RCP4 & 5 & 0.039 & 0.204 & -0.050 & 0.050 & 0.001 \\
\hline
\end{tabular}


Table S18. Molecular graphs and topological parameters of electron density at the $\mathrm{BCP}$ and $\mathrm{RCP}$ for Int.1-F.

\begin{tabular}{ccccccc}
\hline Name & Atoms & Rho & DelSqRho & $\mathbf{V}(\mathbf{r})$ & $\mathbf{G}(\mathbf{r})$ & $\mathbf{H}(\mathbf{r})$ \\
\hline BCP1 & C1 - N2 & 0.365 & -1.194 & -0.706 & 0.204 & -0.502 \\
BCP2 & N2 - S3 & 0.224 & -0.422 & -0.351 & 0.123 & -0.228 \\
BCP3 & S4 - N5 & 0.232 & -0.448 & -0.409 & 0.148 & -0.261 \\
BCP4 & N5 - S8 & 0.013 & 0.045 & -0.008 & 0.010 & 0.002 \\
BCP5 & S3 - S4 & 0.139 & -0.057 & -0.112 & 0.049 & -0.063 \\
BCP6 & N5 - S9 & 0.013 & 0.044 & -0.008 & 0.010 & 0.002 \\
BCP7 & C1 - N5 & 0.356 & -1.145 & -0.665 & 0.190 & -0.476 \\
BCP8 & S8 - S9 & 0.139 & -0.059 & -0.112 & 0.049 & -0.063 \\
BCP9 & C6 - N7 & 0.362 & -1.177 & -0.695 & 0.200 & -0.495 \\
BCP10 & N7 - S8 & 0.224 & -0.427 & -0.354 & 0.124 & -0.230 \\
BCP11 & C6 - N10 & 0.360 & -1.165 & -0.683 & 0.196 & -0.487 \\
BCP12 & S9 - N10 & 0.232 & -0.448 & -0.414 & 0.151 & -0.263 \\
BCP13 & C1 - F11 & 0.294 & -0.200 & -0.851 & 0.401 & -0.450 \\
BCP14 & C6 - F12 & 0.290 & -0.199 & -0.836 & 0.393 & -0.443 \\
& & & & & & \\
\hline Name & Atom Count & Rho & DelSqRho & $\mathbf{V ( r )}$ & $\mathbf{G ( r )}$ & $\mathbf{H ( r )}$ \\
\hline RCP1 & $\mathbf{5}$ & 0.040 & 0.202 & -0.050 & 0.050 & 0.000 \\
RCP2 & $\mathbf{5}$ & 0.040 & 0.203 & -0.050 & 0.050 & 0.000 \\
RCP3 & $\mathbf{3}$ & 0.013 & 0.045 & -0.008 & 0.010 & 0.002 \\
\hline
\end{tabular}

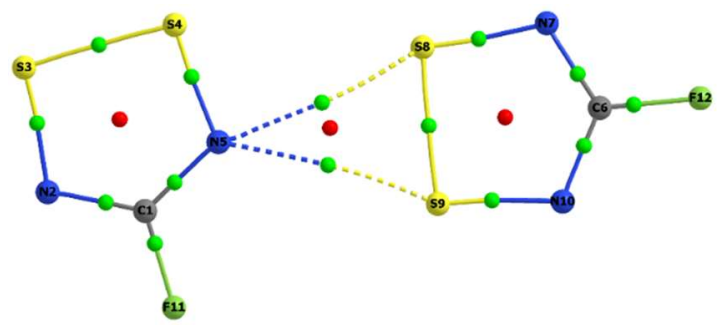


Table S19. Molecular graphs and topological parameters of electron density at the BCP and RCP for Int.2-Cl.

\begin{tabular}{|c|c|c|c|c|c|c|}
\hline Name & Atoms & Rho & DelSqRho & $V(r)$ & $G(r)$ & $H(r)$ \\
\hline BCP1 & $\mathrm{Cl} 1$ - C2 & 0.209 & -0.265 & -0.207 & 0.070 & -0.136 \\
\hline BCP2 & C2 - N3 & 0.358 & -1.145 & -0.707 & 0.210 & -0.497 \\
\hline BCP3 & N3 - S4 & 0.223 & -0.415 & -0.349 & 0.123 & -0.227 \\
\hline BCP4 & C2 - N6 & 0.344 & -1.056 & -0.631 & 0.183 & -0.447 \\
\hline BCP5 & S4 - S5 & 0.138 & -0.053 & -0.112 & 0.049 & -0.062 \\
\hline BCP6 & S5 - N6 & 0.232 & -0.448 & -0.402 & 0.145 & -0.257 \\
\hline BCP7 & N6 - Cl7 & 0.005 & 0.020 & -0.003 & 0.004 & 0.001 \\
\hline BCP8 & S4 - Cl7 & 0.005 & 0.018 & -0.002 & 0.003 & 0.001 \\
\hline BCP9 & S5 - Cl7 & 0.005 & 0.020 & -0.003 & 0.004 & 0.001 \\
\hline ВСР10 & Cl1 - N12 & 0.005 & 0.020 & -0.003 & 0.004 & 0.001 \\
\hline BCP11 & $\mathrm{Cl} 7$ - $\mathrm{C} 8$ & 0.209 & -0.265 & -0.207 & 0.070 & -0.136 \\
\hline BCP12 & Cl1 - S10 & 0.005 & 0.018 & -0.002 & 0.003 & 0.001 \\
\hline ВCP13 & C8 - N9 & 0.358 & -1.145 & -0.707 & 0.210 & -0.497 \\
\hline ВСР14 & N9 - S10 & 0.223 & -0.415 & -0.349 & 0.123 & -0.227 \\
\hline ВCP15 & Cl1 - S11 & 0.005 & 0.020 & -0.003 & 0.004 & 0.001 \\
\hline ВCP16 & C8 - N12 & 0.344 & -1.056 & -0.631 & 0.183 & -0.447 \\
\hline ВCP17 & S10 - S11 & 0.138 & -0.053 & -0.112 & 0.049 & -0.062 \\
\hline ВCР18 & S11 - N12 & 0.232 & -0.448 & -0.402 & 0.145 & -0.257 \\
\hline Name & Atom Count & Rho & DelSqRho & $V(r)$ & $G(r)$ & $H(r)$ \\
\hline RCP1 & 6 & 0.004 & 0.015 & -0.002 & 0.003 & 0.001 \\
\hline $\mathrm{RCP2}$ & 5 & 0.039 & 0.202 & -0.050 & 0.050 & 0.001 \\
\hline RCP3 & 4 & 0.004 & 0.018 & -0.002 & 0.003 & 0.001 \\
\hline RCP4 & 7 & 0.004 & 0.018 & -0.002 & 0.003 & 0.001 \\
\hline RCP5 & 5 & 0.039 & 0.202 & -0.050 & 0.050 & 0.001 \\
\hline RCP6 & 3 & 0.005 & 0.019 & -0.002 & 0.004 & 0.001 \\
\hline RCP7 & 3 & 0.005 & 0.020 & -0.003 & 0.004 & 0.001 \\
\hline RCP8 & 3 & 0.005 & 0.019 & -0.002 & 0.004 & 0.001 \\
\hline RCP9 & 3 & 0.005 & 0.020 & -0.003 & 0.004 & 0.001 \\
\hline
\end{tabular}


Table S20. Molecular graphs and topological parameters of electron density at the BCP and RCP for Int.2-Br.

\begin{tabular}{|c|c|c|c|c|c|c|}
\hline Name & Atoms & Rho & DelSqRho & $V(r)$ & $G(r)$ & $H(r)$ \\
\hline BCP1 & S2 - S3 & 0.138 & -0.053 & -0.112 & 0.049 & -0.062 \\
\hline BCP2 & S2 - N4 & 0.232 & -0.447 & -0.397 & 0.143 & -0.255 \\
\hline BCP3 & S3 - N5 & 0.225 & -0.420 & -0.359 & 0.127 & -0.232 \\
\hline BCP4 & $\mathrm{Br} 1$ - C6 & 0.168 & -0.135 & -0.155 & 0.061 & -0.094 \\
\hline BCP5 & N4 - C6 & 0.338 & -1.020 & -0.611 & 0.178 & -0.433 \\
\hline BCP6 & N5 - C6 & 0.366 & -1.183 & -0.776 & 0.240 & -0.536 \\
\hline BCP7 & N4 - Br7 & 0.006 & 0.021 & -0.003 & 0.004 & 0.001 \\
\hline BCP8 & S3 - Br7 & 0.005 & 0.017 & -0.002 & 0.003 & 0.001 \\
\hline BCP9 & Br1 - N10 & 0.006 & 0.021 & -0.003 & 0.004 & 0.001 \\
\hline BCP10 & Br1 - S9 & 0.005 & 0.017 & -0.002 & 0.003 & 0.001 \\
\hline BCP11 & S8 - S9 & 0.138 & -0.053 & -0.112 & 0.049 & -0.062 \\
\hline BCP12 & S8 - N10 & 0.232 & -0.448 & -0.398 & 0.143 & -0.255 \\
\hline ВCP13 & S9 - N11 & 0.225 & -0.420 & -0.359 & 0.127 & -0.232 \\
\hline ВCP14 & Br7 - C12 & 0.168 & -0.135 & -0.155 & 0.061 & -0.094 \\
\hline BCP15 & N10 - C12 & 0.338 & -1.020 & -0.611 & 0.178 & -0.433 \\
\hline BCP16 & N11 - C12 & 0.366 & -1.182 & -0.775 & 0.240 & -0.535 \\
\hline Name & Atom Count & Rho & DelSqRho & $V(r)$ & $G(r)$ & $H(r)$ \\
\hline RCP1 & 5 & 0.040 & 0.205 & -0.050 & 0.051 & 0.001 \\
\hline $\mathrm{RCP2}$ & 6 & 0.004 & 0.014 & -0.002 & 0.003 & 0.001 \\
\hline $\mathrm{RCP} 3$ & 5 & 0.040 & 0.205 & -0.050 & 0.051 & 0.001 \\
\hline RCP4 & 4 & 0.005 & 0.017 & -0.002 & 0.003 & 0.001 \\
\hline RCP5 & 5 & 0.004 & 0.017 & -0.002 & 0.003 & 0.001 \\
\hline RCP6 & 4 & 0.005 & 0.017 & -0.002 & 0.003 & 0.001 \\
\hline $\mathrm{RCP7}$ & 5 & 0.004 & 0.017 & -0.002 & 0.003 & 0.001 \\
\hline
\end{tabular}


Table S21. Molecular graphs and topological parameters of electron density at the BCP and RCP for Int.2-F.

\begin{tabular}{ccccccc}
\hline Name & Atoms & Rho & DelSqRho & V(r) & G(r) & H(r) \\
\hline BCP1 & C1 - N2 & 0.361 & -1.165 & -0.690 & 0.199 & -0.490 \\
BCP2 & N2 - S3 & 0.223 & -0.416 & -0.355 & 0.125 & -0.229 \\
BCP3 & S3 - S4 & 0.138 & -0.054 & -0.112 & 0.049 & -0.063 \\
BCP4 & C1 - N5 & 0.347 & -1.066 & -0.617 & 0.175 & -0.442 \\
BCP5 & C1 - F11 & 0.003 & 0.014 & -0.002 & 0.003 & 0.001 \\
BCP6 & S4 - N5 & 0.232 & -0.443 & -0.408 & 0.149 & -0.259 \\
BCP7 & C6 - F12 & 0.003 & 0.014 & -0.002 & 0.003 & 0.001 \\
BCP8 & C6 - N7 & 0.361 & -1.165 & -0.690 & 0.199 & -0.490 \\
BCP9 & N7 - S8 & 0.223 & -0.416 & -0.355 & 0.125 & -0.229 \\
BCP10 & S8 - S9 & 0.138 & -0.054 & -0.112 & 0.049 & -0.063 \\
BCP11 & C6 - N10 & 0.347 & -1.066 & -0.617 & 0.175 & -0.442 \\
BCP12 & S9 - N10 & 0.232 & -0.443 & -0.408 & 0.149 & -0.259 \\
BCP13 & C6 - F11 & 0.270 & -0.335 & -0.713 & 0.315 & -0.398 \\
BCP14 & C1 - F12 & 0.270 & -0.335 & -0.713 & 0.315 & -0.398 \\
& & & & & & \\
\hline Name & Atom Count & Rho & DelSqRho & $\mathbf{V ( r )}$ & $\mathbf{G ( r )}$ & $\mathbf{H ( r )}$ \\
\hline RCP1 & $\mathbf{5}$ & 0.040 & 0.200 & -0.049 & 0.050 & 0.000 \\
RCP2 & $\mathbf{4}$ & 0.003 & 0.013 & -0.001 & 0.002 & 0.001 \\
RCP3 & $\mathbf{5}$ & 0.040 & 0.200 & -0.049 & 0.050 & 0.000 \\
\hline
\end{tabular}

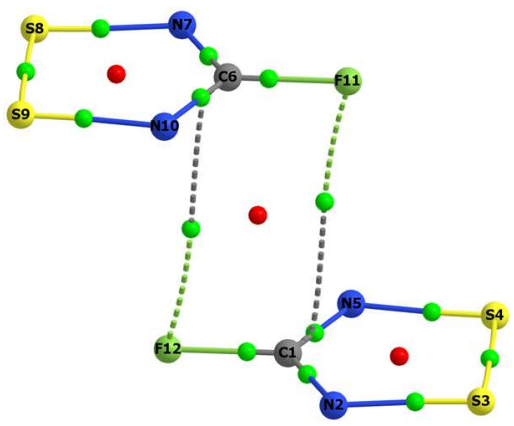


Table S22. Molecular graphs and topological parameters of electron density at the $\mathrm{BCP}$ and $\mathrm{RCP}$ for Int.3-Cl.

\begin{tabular}{ccccccc}
\hline Name & Atoms & Rho & DelSqRho & V(r) & G(r) & H(r) \\
\hline BCP1 & S1 - S2 & 0.137 & -0.053 & -0.111 & 0.049 & -0.062 \\
BCP2 & N4 - C6 & 0.355 & -1.126 & -0.682 & 0.200 & -0.482 \\
BCP3 & N5 - N11 & 0.011 & 0.044 & -0.007 & 0.009 & 0.002 \\
BCP4 & N5 - C6 & 0.359 & -1.156 & -0.711 & 0.211 & -0.500 \\
BCP5 & S2 - N5 & 0.230 & -0.443 & -0.391 & 0.140 & -0.251 \\
BCP6 & S1 - N4 & 0.225 & -0.422 & -0.354 & 0.124 & -0.230 \\
BCP7 & C13 - C6 & 0.207 & -0.256 & -0.202 & 0.069 & -0.133 \\
BCP8 & N5 - S8 & 0.011 & 0.037 & -0.007 & 0.008 & 0.001 \\
BCP9 & S7 - S8 & 0.137 & -0.053 & -0.111 & 0.049 & -0.062 \\
BCP10 & N10 - C12 & 0.355 & -1.126 & -0.682 & 0.200 & -0.482 \\
BCP11 & N11 - C12 & 0.359 & -1.156 & -0.711 & 0.211 & -0.500 \\
BCP12 & S8 - N11 & 0.230 & -0.443 & -0.391 & 0.140 & -0.251 \\
BCP13 & S7 - N10 & 0.225 & -0.422 & -0.354 & 0.124 & -0.230 \\
BCP14 & C19 - C12 & 0.207 & -0.256 & -0.202 & 0.069 & -0.133 \\
BCP15 & S2 - N11 & 0.011 & 0.037 & -0.007 & 0.008 & 0.001 \\
& & & & & & \\
\hline Name & Atom Count & Rho & DelSqRho & V(r) & $\mathbf{G ( r )}$ & H(r) \\
\hline RCP1 & $\mathbf{3}$ & 0.011 & 0.040 & -0.007 & 0.008 & 0.002 \\
RCP2 & $\mathbf{5}$ & 0.040 & 0.203 & -0.050 & 0.050 & 0.000 \\
RCP3 & $\mathbf{3}$ & 0.011 & 0.040 & -0.007 & 0.008 & 0.002 \\
RCP4 & $\mathbf{5}$ & 0.040 & 0.203 & -0.050 & 0.050 & 0.000 \\
\hline & & & & & &
\end{tabular}

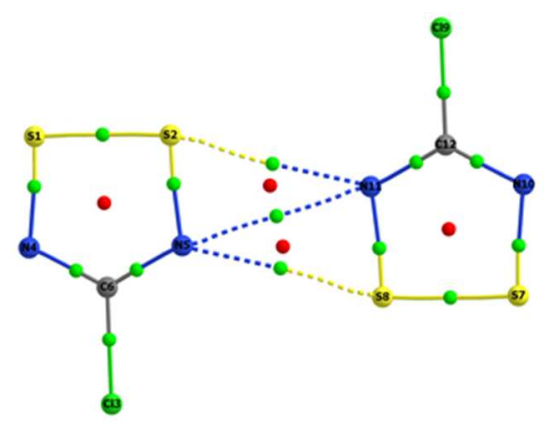


Table S23. Molecular graphs and topological parameters of electron density at the $\mathrm{BCP}$ and $\mathrm{RCP}$ for Int.3-Br.

\begin{tabular}{|c|c|c|c|c|c|c|}
\hline Name & Atoms & Rho & DelSqRho & $V(r)$ & $G(r)$ & $H(r)$ \\
\hline BCP1 & S1 - S2 & 0.137 & -0.053 & -0.111 & 0.049 & -0.062 \\
\hline BCP2 & S2 - N4 & 0.230 & -0.440 & -0.389 & 0.139 & -0.249 \\
\hline BCP3 & S1 - N3 & 0.224 & -0.419 & -0.353 & 0.124 & -0.229 \\
\hline BCP4 & N3 - C5 & 0.357 & -1.140 & -0.710 & 0.213 & -0.498 \\
\hline BCP5 & N4 - N9 & 0.011 & 0.044 & -0.007 & 0.009 & 0.002 \\
\hline BCP6 & N4 - C5 & 0.354 & -1.122 & -0.691 & 0.205 & -0.486 \\
\hline BCP7 & N4 - S7 & 0.011 & 0.037 & -0.007 & 0.008 & 0.001 \\
\hline BCP8 & S6 - S7 & 0.137 & -0.053 & -0.111 & 0.049 & -0.062 \\
\hline BCP9 & S7 - N9 & 0.230 & -0.440 & -0.389 & 0.139 & -0.249 \\
\hline BCP10 & S6 - N8 & 0.224 & -0.419 & -0.353 & 0.124 & -0.229 \\
\hline BCP11 & S2 - N9 & 0.011 & 0.037 & -0.007 & 0.008 & 0.001 \\
\hline BCP12 & N8 - C10 & 0.357 & -1.140 & -0.710 & 0.213 & -0.498 \\
\hline BCP13 & N9 - C10 & 0.354 & -1.122 & -0.691 & 0.205 & -0.486 \\
\hline BCP14 & C5 - Br11 & 0.164 & -0.125 & -0.146 & 0.058 & -0.089 \\
\hline BCP15 & C10 - Br12 & 0.164 & -0.125 & -0.146 & 0.058 & -0.089 \\
\hline Name & Atom Count & Rho & DelSqRho & $V(r)$ & $G(r)$ & $H(r)$ \\
\hline RCP1 & 3 & 0.011 & 0.040 & -0.007 & 0.009 & 0.002 \\
\hline RCP2 & 5 & 0.040 & 0.204 & -0.050 & 0.050 & 0.000 \\
\hline RCP3 & 3 & 0.011 & 0.040 & -0.007 & 0.009 & 0.002 \\
\hline RCP4 & 5 & 0.040 & 0.204 & -0.050 & 0.050 & 0.000 \\
\hline
\end{tabular}


Table S24. Molecular graphs and topological parameters of electron density at the $\mathrm{BCP}$ and $\mathrm{RCP}$ for Int.3-F.

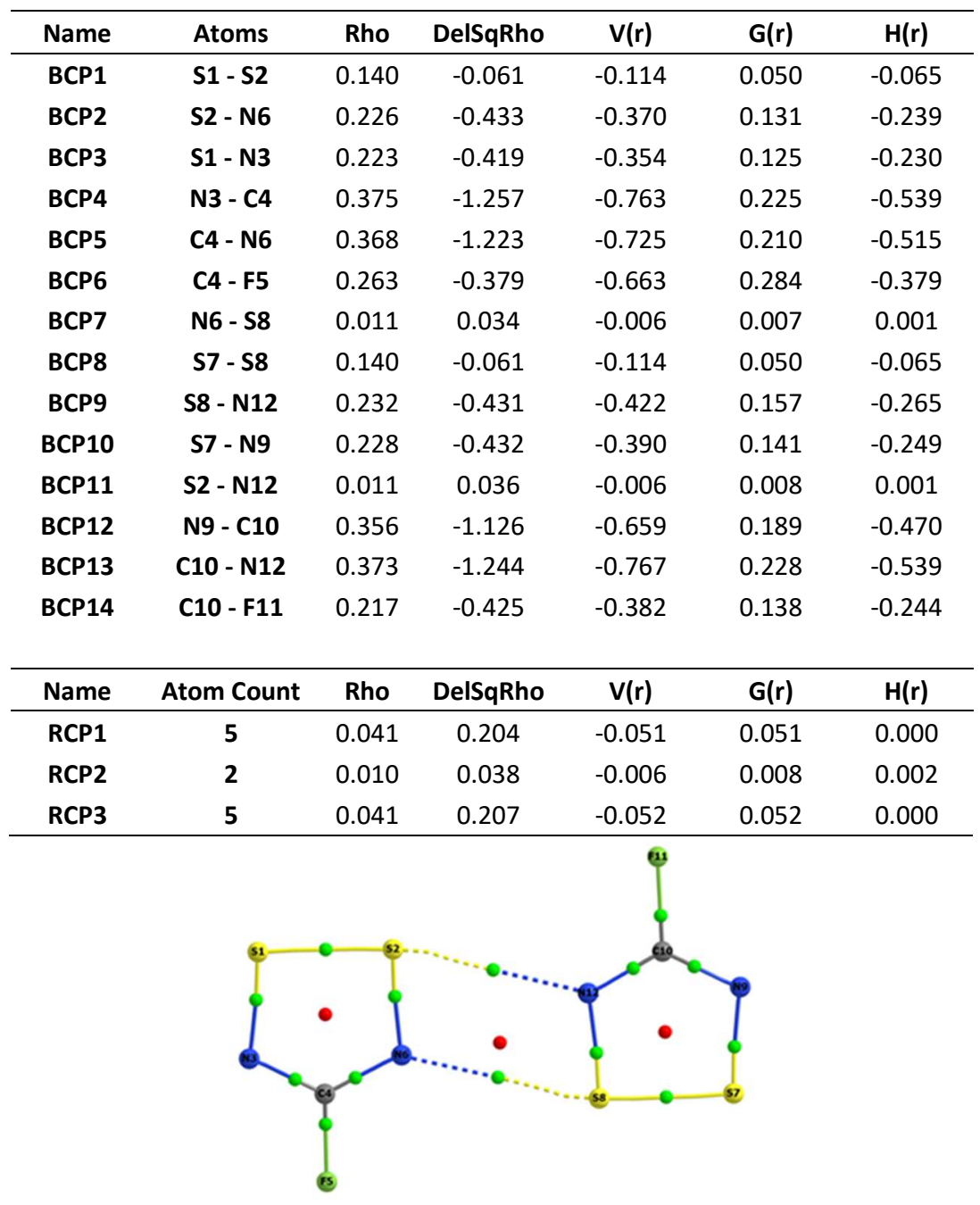


Table S25. Molecular graphs and topological parameters of electron density at the BCP and RCP for Int.4-Cl.

\begin{tabular}{|c|c|c|c|c|c|c|}
\hline Name & Atoms & Rho & DelSqRho & $V(r)$ & $G(r)$ & $H(r)$ \\
\hline BCP1 & $\mathrm{Cl} 1-\mathrm{C} 2$ & 0.210 & -0.261 & -0.207 & 0.071 & -0.136 \\
\hline BCP2 & $\mathrm{C} 2-\mathrm{N} 6$ & 0.344 & -1.059 & -0.633 & 0.184 & -0.449 \\
\hline BCP3 & $\mathrm{C} 2-\mathrm{N} 3$ & 0.358 & -1.147 & -0.710 & 0.212 & -0.498 \\
\hline BCP4 & S4 - S5 & 0.138 & -0.053 & -0.112 & 0.049 & -0.062 \\
\hline BCP5 & N3 - S4 & 0.224 & -0.416 & -0.348 & 0.122 & -0.226 \\
\hline BCP6 & S5 - N6 & 0.232 & -0.451 & -0.399 & 0.143 & -0.256 \\
\hline BCP7 & $\mathrm{Cl} 1-\mathrm{N} 9$ & 0.009 & 0.031 & -0.005 & 0.006 & 0.001 \\
\hline BCP8 & $\mathrm{Cl} 7-\mathrm{C} 8$ & 0.209 & -0.262 & -0.207 & 0.071 & -0.136 \\
\hline BCP9 & $\mathrm{C} 8-\mathrm{N} 9$ & 0.358 & -1.146 & -0.707 & 0.210 & -0.497 \\
\hline BCP10 & $\mathrm{C} 8-\mathrm{N} 12$ & 0.344 & -1.058 & -0.631 & 0.183 & -0.448 \\
\hline BCP11 & N9 - S10 & 0.223 & -0.415 & -0.349 & 0.123 & -0.226 \\
\hline BCP12 & $\mathrm{S} 11-\mathrm{N} 12$ & 0.232 & -0.449 & -0.400 & 0.144 & -0.256 \\
\hline BCP13 & S10 - S11 & 0.138 & -0.053 & -0.112 & 0.049 & -0.062 \\
\hline Name & Atom Count & Rho & DelSqRho & $V(r)$ & $G(r)$ & $H(r)$ \\
\hline RCP1 & 5 & 0.039 & 0.203 & -0.050 & 0.050 & 0.001 \\
\hline $\mathrm{RCP2}$ & 5 & 0.039 & 0.203 & -0.050 & 0.050 & 0.001 \\
\hline
\end{tabular}


Table S26. Molecular graphs and topological parameters of electron density at the $\mathrm{BCP}$ and $\mathrm{RCP}$ for Int.4-Br.

\begin{tabular}{|c|c|c|c|c|c|c|}
\hline Name & Atoms & Rho & DelSqRho & $V(r)$ & $G(r)$ & $H(r)$ \\
\hline BCP1 & Br1 - C6 & 0.169 & -0.132 & -0.158 & 0.062 & -0.095 \\
\hline BCP2 & S2 - S3 & 0.138 & -0.052 & -0.112 & 0.049 & -0.062 \\
\hline BCP3 & S2 - N4 & 0.232 & -0.450 & -0.395 & 0.141 & -0.254 \\
\hline BCP4 & N4 - C6 & 0.338 & -1.024 & -0.614 & 0.179 & -0.435 \\
\hline BCP5 & N5 - C6 & 0.366 & -1.181 & -0.779 & 0.242 & -0.537 \\
\hline BCP6 & S3 - N5 & 0.225 & -0.420 & -0.357 & 0.126 & -0.231 \\
\hline BCP7 & Br1 - N11 & 0.011 & 0.038 & -0.007 & 0.008 & 0.002 \\
\hline BCP8 & S8 - S9 & 0.138 & -0.053 & -0.112 & 0.049 & -0.062 \\
\hline BCP9 & $\mathrm{Br} 7$ - C12 & 0.168 & -0.133 & -0.156 & 0.061 & -0.094 \\
\hline BCP10 & S8 - N10 & 0.232 & -0.449 & -0.396 & 0.142 & -0.254 \\
\hline BCP11 & S9 - N11 & 0.224 & -0.419 & -0.359 & 0.127 & -0.232 \\
\hline BCP12 & N11 - C12 & 0.366 & -1.183 & -0.776 & 0.240 & -0.536 \\
\hline BCP13 & N10 - C12 & 0.339 & -1.022 & -0.612 & 0.178 & -0.434 \\
\hline Name & Atom Count & Rho & DelSqRho & $V(r)$ & $G(r)$ & $H(r)$ \\
\hline RCP1 & 5 & 0.040 & 0.205 & -0.050 & 0.051 & 0.001 \\
\hline $\mathrm{RCP2}$ & 5 & 0.040 & 0.205 & -0.050 & 0.051 & 0.001 \\
\hline
\end{tabular}


Table S27. Molecular graphs and topological parameters of electron density at the BCP and RCP for Int.4-F.

\begin{tabular}{ccccccc}
\hline Name & Atoms & Rho & DelSqRho & V(r) & $\mathbf{G ( r )}$ & $\mathbf{H}(\mathbf{r})$ \\
\hline BCP1 & C1 - N2 & 0.360 & -1.205 & -0.695 & 0.197 & -0.498 \\
BCP2 & N2 - S3 & 0.223 & -0.425 & -0.353 & 0.123 & -0.229 \\
BCP3 & C1 - F11 & 0.293 & -0.241 & -0.841 & 0.390 & -0.451 \\
BCP4 & C1 - N5 & 0.359 & -1.202 & -0.694 & 0.197 & -0.497 \\
BCP5 & S4 - N5 & 0.231 & -0.449 & -0.408 & 0.148 & -0.260 \\
BCP6 & S3 - S4 & 0.138 & -0.056 & -0.111 & 0.048 & -0.062 \\
BCP7 & N7 - F11 & 0.004 & 0.017 & -0.003 & 0.004 & 0.001 \\
BCP8 & C6 - N7 & 0.361 & -1.212 & -0.701 & 0.199 & -0.502 \\
BCP9 & N7 - S8 & 0.223 & -0.425 & -0.352 & 0.123 & -0.229 \\
BCP10 & S9 - N10 & 0.231 & -0.449 & -0.409 & 0.148 & -0.261 \\
BCP11 & S8 - S9 & 0.138 & -0.057 & -0.111 & 0.048 & -0.062 \\
BCP12 & C6 - N10 & 0.359 & -1.203 & -0.694 & 0.197 & -0.498 \\
BCP13 & C6 - F12 & 0.291 & -0.233 & -0.831 & 0.387 & -0.445 \\
& & & & & & \\
\hline Name & Atom Count & Rho & DelSqRho & $\mathbf{V ( r )}$ & $\mathbf{G ( r )}$ & $\mathbf{H}(\mathbf{r})$ \\
\hline RCP1 & $\mathbf{5}$ & 0.040 & 0.200 & -0.050 & 0.050 & 0.000 \\
RCP2 & $\mathbf{5}$ & 0.040 & 0.200 & -0.050 & 0.050 & 0.000 \\
\hline
\end{tabular}

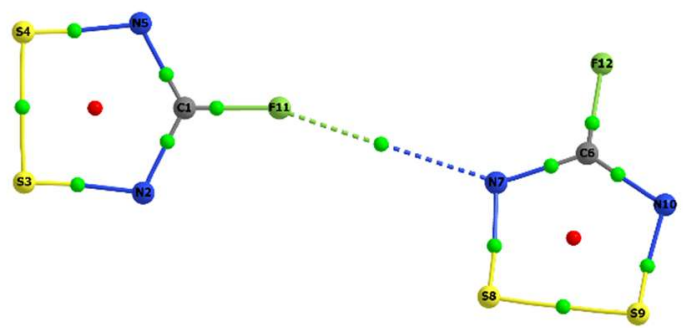


Table S28. Molecular graphs and topological parameters of electron density at the BCP and RCP for Int.5-Cl.

\begin{tabular}{|c|c|c|c|c|c|c|}
\hline Name & Atoms & Rho & DelSqRho & $V(r)$ & $G(r)$ & $H(r)$ \\
\hline BCP1 & $\mathrm{Cl} 1$ - Cl7 & 0.006 & 0.022 & -0.003 & 0.004 & 0.001 \\
\hline BCP2 & $\mathrm{Cl} 1$ - C2 & 0.209 & -0.262 & -0.207 & 0.071 & -0.136 \\
\hline ВСР3 & C2 - N3 & 0.358 & -1.147 & -0.708 & 0.211 & -0.498 \\
\hline BCP4 & N3 - S4 & 0.223 & -0.415 & -0.348 & 0.122 & -0.226 \\
\hline BCP5 & C2 - N6 & 0.344 & -1.058 & -0.632 & 0.184 & -0.448 \\
\hline BCP6 & S5 - N6 & 0.232 & -0.450 & -0.400 & 0.144 & -0.256 \\
\hline BCP7 & S4 - S5 & 0.138 & -0.053 & -0.112 & 0.049 & -0.062 \\
\hline ВСР8 & $\mathrm{Cl} 7-\mathrm{C} 8$ & 0.209 & -0.262 & -0.207 & 0.071 & -0.136 \\
\hline ВCP9 & C8 - N9 & 0.358 & -1.147 & -0.708 & 0.211 & -0.498 \\
\hline BCP10 & N9 - S10 & 0.223 & -0.415 & -0.348 & 0.122 & -0.226 \\
\hline BCP11 & C8 - N12 & 0.344 & -1.058 & -0.632 & 0.184 & -0.448 \\
\hline BCP12 & S11 - N12 & 0.232 & -0.450 & -0.400 & 0.144 & -0.256 \\
\hline BCP13 & S10 - S11 & 0.138 & -0.053 & -0.112 & 0.049 & -0.062 \\
\hline Name & Atom Count & Rho & DelSqRho & $V(r)$ & $G(r)$ & $H(r)$ \\
\hline RCP1 & 5 & 0.039 & 0.203 & -0.050 & 0.050 & 0.001 \\
\hline RCP2 & 5 & 0.039 & 0.203 & -0.050 & 0.050 & 0.001 \\
\hline
\end{tabular}


Table S29. Molecular graphs and topological parameters of electron density at the BCP and RCP for Int.5-Br.

\begin{tabular}{ccccccc}
\hline Name & Atoms & Rho & DelSqRho & V(r) & G(r) & H(r) \\
\hline BCP1 & S2 - S3 & 0.138 & -0.052 & -0.112 & 0.049 & -0.062 \\
BCP2 & S2 - N4 & 0.232 & -0.449 & -0.396 & 0.142 & -0.254 \\
BCP3 & Br1 - Br7 & 0.007 & 0.022 & -0.003 & 0.004 & 0.001 \\
BCP4 & S3 - N5 & 0.225 & -0.420 & -0.358 & 0.126 & -0.231 \\
BCP5 & N5 - C6 & 0.366 & -1.182 & -0.777 & 0.241 & -0.536 \\
BCP6 & Br1 - C6 & 0.168 & -0.132 & -0.156 & 0.061 & -0.094 \\
BCP7 & N4 - C6 & 0.338 & -1.022 & -0.612 & 0.178 & -0.434 \\
BCP8 & S8 - S9 & 0.138 & -0.052 & -0.112 & 0.049 & -0.062 \\
BCP9 & S8 - N10 & 0.232 & -0.449 & -0.396 & 0.142 & -0.254 \\
BCP10 & S9 - N11 & 0.225 & -0.420 & -0.358 & 0.126 & -0.231 \\
BCP11 & N11 - C12 & 0.366 & -1.182 & -0.777 & 0.241 & -0.536 \\
BCP12 & Br7 - C12 & 0.168 & -0.132 & -0.156 & 0.061 & -0.094 \\
BCP13 & N10 - C12 & 0.338 & -1.022 & -0.612 & 0.178 & -0.434 \\
& & & & & & \\
\hline Name & Atom Count & Rho & DelSqRho & $\mathbf{V ( r )}$ & $\mathbf{G ( r )}$ & $\mathbf{H}(\mathbf{r})$ \\
\hline RCP1 & $\mathbf{5}$ & 0.040 & 0.205 & -0.050 & 0.051 & 0.001 \\
RCP2 & $\mathbf{5}$ & 0.040 & 0.205 & -0.050 & 0.051 & 0.001 \\
\hline
\end{tabular}

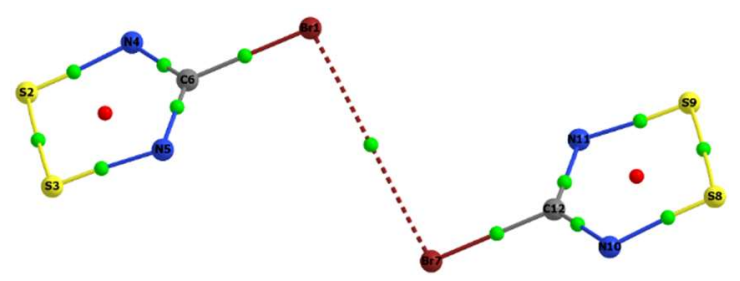


Table S30. Molecular graphs and topological parameters of electron density at the BCP and RCP for Int.6-CN.

\begin{tabular}{|c|c|c|c|c|c|c|}
\hline Name & Atoms & Rho & DelSqRho & $V(r)$ & $G(r)$ & $H(r)$ \\
\hline BCP1 & S1 - S2 & 0.137 & -0.069 & -0.113 & 0.048 & -0.065 \\
\hline BCP2 & $\mathrm{C} 6-\mathrm{C} 7$ & 0.247 & -0.601 & -0.262 & 0.056 & -0.206 \\
\hline BCP3 & S2 - N12 & 0.012 & 0.043 & -0.008 & 0.009 & 0.002 \\
\hline BCP4 & S2 - N3 & 0.224 & -0.189 & -0.504 & 0.228 & -0.275 \\
\hline BCP5 & S1 - N4 & 0.246 & 0.064 & -0.635 & 0.326 & -0.309 \\
\hline BCP6 & N5 - C7 & 0.539 & 0.326 & -2.157 & 1.119 & -1.038 \\
\hline BCP7 & N3 - C6 & 0.396 & -0.792 & -1.092 & 0.447 & -0.645 \\
\hline BCP8 & N4 - C6 & 0.321 & -0.904 & -0.594 & 0.184 & -0.410 \\
\hline BCP9 & C13 - C14 & 0.247 & -0.604 & -0.263 & 0.056 & -0.207 \\
\hline BCP10 & $58-59$ & 0.137 & -0.065 & -0.113 & 0.049 & -0.065 \\
\hline BCP11 & N10 - C13 & 0.396 & -0.789 & -1.093 & 0.448 & -0.645 \\
\hline BCP12 & S9 - N10 & 0.224 & -0.208 & -0.498 & 0.223 & -0.275 \\
\hline ВCP13 & N11 - C13 & 0.321 & -0.904 & -0.594 & 0.184 & -0.410 \\
\hline ВCP14 & S8 - N11 & 0.245 & 0.045 & -0.630 & 0.321 & -0.309 \\
\hline BCP15 & N12 - C14 & 0.538 & 0.324 & -2.144 & 1.112 & -1.032 \\
\hline BCP16 & S1 - N12 & 0.012 & 0.043 & -0.008 & 0.009 & 0.002 \\
\hline Name & Atom Count & Rho & DelSqRho & $V(r)$ & $G(r)$ & $H(r)$ \\
\hline RCP1 & 3 & 0.011 & 0.045 & -0.007 & 0.009 & 0.002 \\
\hline $\mathrm{RCP2}$ & 5 & 0.037 & 0.218 & -0.043 & 0.049 & 0.006 \\
\hline RCP3 & 5 & 0.037 & 0.219 & -0.043 & 0.049 & 0.006 \\
\hline
\end{tabular}

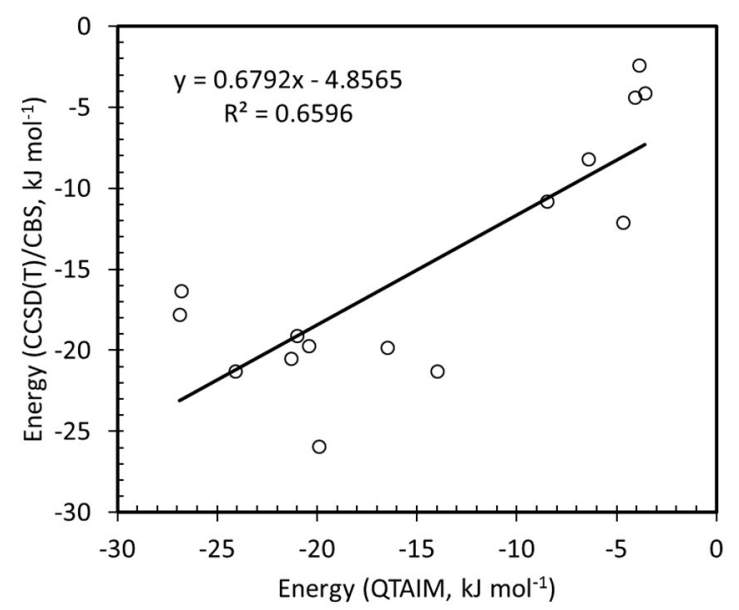

Figure S1. Plot of benchmark vs QTAIM interaction energies $\left(\mathrm{kJ} \mathrm{mol}^{-1}\right)$ for the intermolecular interactions presented in Figure 3. 